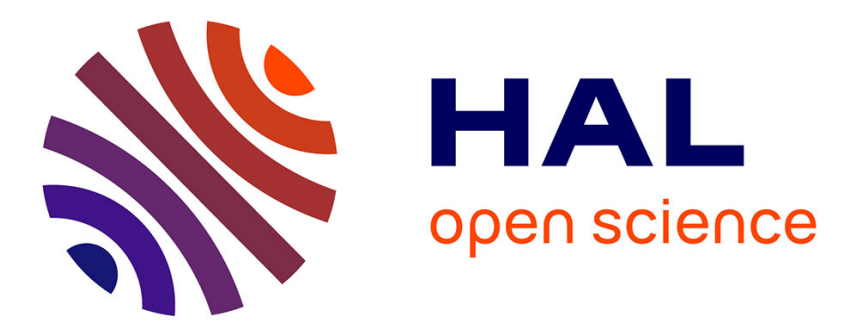

\title{
Interfacial reaction rates and free energy of cubic clusters
}

Joel Lepinoux

\section{To cite this version:}

Joel Lepinoux. Interfacial reaction rates and free energy of cubic clusters. Philosophical Magazine, 2004, 85 (30), pp.3585. 10.1080/14786430500228648 . hal-00513559

\section{HAL Id: hal-00513559 \\ https://hal.science/hal-00513559}

Submitted on 1 Sep 2010

HAL is a multi-disciplinary open access archive for the deposit and dissemination of scientific research documents, whether they are published or not. The documents may come from teaching and research institutions in France or abroad, or from public or private research centers.
L'archive ouverte pluridisciplinaire HAL, est destinée au dépôt et à la diffusion de documents scientifiques de niveau recherche, publiés ou non, émanant des établissements d'enseignement et de recherche français ou étrangers, des laboratoires publics ou privés. 


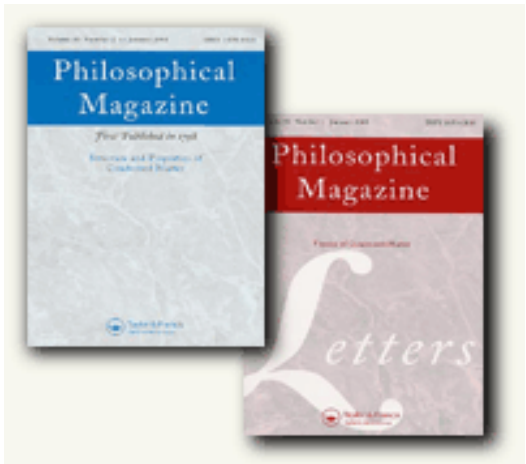

\section{Interfacial reaction rates and free energy of cubic clusters}

\begin{tabular}{|r|l|}
\hline Journal: & Philosophical Magazine \& Philosophical Magazine Letters \\
\hline Manuscript ID: & TPHM-05-Apr-0113 \\
\hline Journal Selection: & Philosophical Magazine \\
\hline Date Submitted by the \\
Author: & 22-Apr-2005 \\
\hline Complete List of Authors: & Lepinoux, Joel; LTPCM, INPG \\
\hline Keywords: & clusters, computation, Monte-Carlo, precipitation \\
\hline Keywords (user supplied): & cluster dynamics, free energy \\
\hline &
\end{tabular}

\section{今 ScholarONE" \\ Manuscript Central}




\begin{abstract}
A new formulation of interfacial reaction rates for clusters in binary alloys is presented. It accounts for the matrix structure and the topological properties of cluster at the atomic scale. It is shown that the probabilities per unit time that a solute atom be captured or released by a cluster are functions of the partition function but also of a transition function. The principles of calculation of these functions are general but only the case of cubic clusters is treated here (results can be used for $\mathrm{L}_{12}$ clusters in f.c.c. matrices). Calculations has been done exactly for small clusters $($ size $<10)$, then following a Monte Carlo sampling method at intermediate size as a function of temperature and the interaction energy (a material characteristic). Finally, it is shown that generic results can be extrapolated at higher cluster size in a large range of temperature and / or interaction energy.
\end{abstract}

Keywords: Clusters, Cluster Dynamics, Precipitation, Free Energy, Monte Carlo

\title{
$\S$ 1. INTRODUCTION
}

Cluster dynamics is a simple and elegant numerical method mostly developed by Binder and co-workers (Binder et al. 1974, Binder and Stauffer 1976, Binder 1977) to predict nucleation and growth of clusters in a solid solution. For complete reviews on this field see for instance Martin (1978), Wagner and Kampmann (1991). Martin (to be published) has recently revisited the classical theory of nucleation and its links with cluster dynamics. The foundations of cluster dynamics are briefly recalled in appendix A of the present paper. An interesting feature of this method is that the fundamental equations can be used to describe clusters composed of solute atoms as obtained in segregation (Mathon et al. 1997) or electrodeposition (Berthier et al. 2004), or point defects in irradiated materials (Christien and Barbu 2004). Generally speaking, the simplicity and the versatility of this technique make it a popular alternative for treating situations that are difficult to address with standard mean field methods or with atomistic methods. Note that although in materials science cluster dynamics is mostly used to model precipitation phenomena, it can be seen as a particular application of a more general method, say "class dynamics" (i.e. where each class is a multiple of the unit class), likely to be applied to a wide range of situations in various fields.

The specificity of the cluster dynamics addressed here is the definition of the coefficients involved in the master equation. In precipitation, the evolution of clusters is controlled by two very different mechanisms: interfacial exchanges between the clusters and the surrounding matrix and long distance diffusion (here long distance means a distance of the order of the half distance between clusters, much larger than the cluster size). There are mainly two distinct methods for combining these effects in the literature:

(i) the classical one, often based on the Waite model (1958), using only one pair of coefficients for a given size of cluster (e.g. Clouet et al. 2005), 
(ii) a recent one, proposed by Kelton (2000), using two pairs of coefficients, one for exchanges between the clusters and the surrounding matrix (i.e. the well-defined zone around a cluster where atoms can be captured or released, referred as the "shell" in Kelton's work) and another pair for exchanges between the shell and the rest of the matrix. One of the main interests of the latter formalism is that it clearly separates both mechanisms allowing them to be treated independently. However, apart from this difference in the treatment of the coupling between the short range and the long range effects, the physical basis of the approach proposed by Kelton are the same than those of earlier classical works (e.g. Kelton et al. 1983), in particular the way to account for the free energy of clusters in interfacial exchange rates. A common concern in the literature is precisely that the description of free energy based on the capillary approximation is correct for large clusters but it is obviously too rough an approximation for small clusters. In addition all clusters are taken spherical whatever their size, the lattice structure or the temperature.

Thus the main goal of this paper is to propose a more rigorous definition of these interfacial exchange rates, by examining in detail the processes of capture and release of solute atoms by clusters, at the atomic scale. In order to get the values of these coefficients two computing methods have been developed:

- an exact method which gives the solution in a polynomial form but which can be applied only to small clusters ( $<10$ atoms)

- a Monte Carlo method which can be applied to medium size clusters (few hundreds atoms), giving accurate results but for a given material each run is related to a given temperature. Thus, in order to build a generic solution several runs are necessary at various temperatures then results have to be fitted to be extrapolated at higher sizes and interpolated at other temperatures in the explored range.

Although the model and the calculation procedures can be applied to any crystallographic structures, for the sake of simplicity, the present paper focus on the simple cubic structure but results can be applied to $\mathrm{L}_{12}$ clusters;

\section{§ 2. DEFINITIONS AND NOTATIONS}

This section aims at defining step by step the notations used in the following of this work. The situation considered is a binary system composed of $N_{b}$ solute atoms $B$ in a matrix A $\left(N_{a}+N_{b}=\right.$ constant $)$ and interaction energies limited to first neighbours. With $E_{a}, E_{b b}$ and $E_{a b}$ the pair energies, $Z$ the coordination of the lattice, the potential energy of the system writes:

$W=Z \frac{N_{A}}{2} E_{a a}+Z \frac{N_{B}}{2} E_{b b}+\frac{N_{A B}}{2} W_{a b}$

$W_{a b}$ is the interaction energy parameter defined as:

$W_{a b}=2 E_{a b}-\left(E_{a a}+E_{b b}\right)$

Now considering a given arrangement of the $N_{b}$ solute atoms, their energy can be defined by reference to the equivalent combination of pure media $A$ and $B$ as:

$U_{n}=\left(W_{n}-W_{0}\right)=\frac{1}{2}\left[N_{a b} W_{a b}\right]$ 


\section{§.3. INTERFACIAL REACTION RATES}

Note that equations (5-8) apply also to ordered clusters provided that interactions are limited to second neighbours. In this situation, the interaction terms $W_{a b}$ and $N_{a b}$ deal with second neighbours instead of first neighbours.

\subsection{What is a cluster?}

First of all it is necessary to clearly define what a cluster is (figure 1). A cluster of size $n$ is obviously based on a core of $n$ connected $B$ atoms. But if this usual definition is sufficient 
for approaches considering space as a continuum, it is inappropriate if one wants to examine the reaction processes at the atomic scale. Indeed, if the number of connected $B$ atoms has a well-defined value it necessarily implies that these $B$ atoms are surrounded by an exclusion layer of $A$ atoms, further referred to as the first shell. Each site of this first shell is linked to the cluster by at least one bond. An important consequence of this definition is that there is no direct interaction between a cluster and other $B$ atoms in the matrix. Another consequence is that small size clusters are mainly constituted of their first shell, i.e. the role of their "surface" (constituted of $N_{a b}\{100\}$ facets) is considerably enhanced. When the cluster size increases some $A$ cavities, i.e. local deviations to stoechiometry, can be present. Of course, such sites do not participate in the exchanges between the cluster and the matrix. This definition is still incomplete to address dynamical evolution of clusters; for that, it is necessary to surround again the cluster by a second layer, which will be referred to as the second shell. This most outer shell is formed by the first neighbours of the first shell not belonging to the cluster core. If the nature of the atom occupying a given site of the first shell changes from $A$ to $B$, the considered site becomes immediately part either of the second shell if previously part of the cluster core (i.e. it is a release event) or of the cluster if previously part of the second shell (i.e. it is a capture event). Thus, each event of absorption or emission modifies the local configuration of both shells. Note that figure 1 is a simplified representation of a cluster; in fact, to perform calculations presented in section 5 it is necessary to examine the cluster topology in more details and distinguish various status of atomic sites. However these considerations as well as other technical details are not essential for the present paper and will be reported elsewhere.

- insert figure 1 about here -

\subsection{Changes induced by transitions}

The solute concentrations in the second shell around a cluster and in the rest of the matrix are obviously of the same order of magnitude, then for dilute solutions the probability to find a solute atom in the second shell of a cluster is rather low except for macroscopic sizes. By consequence, it is reasonable to neglect possible interactions between such atoms and other solute atoms located either in the matrix or in the same shell. Thus the probability that a cluster captures and releases a solute atom can be decomposed into two independent contributions: (i) one related to the change of energy and (ii) one related to the change of entropy of its second shell, as proposed by Kelton (2000). This section aims at better defining the energy contribution; the entropy contribution is needed only for coupling interfacial reactions with the long distance diffusion to build a complete model of cluster dynamics and will be presented in a next paper. To make this distinction clearer, in the present paper, the terms capture and release will be preferred instead of the terms emission and absorption, respectively, which refer to the complete processes.

Considering the change of energy arising from the capture or the release of an atom by a cluster simply means comparing two states of the micro-system constituted of $n+1$ solute atoms either in the form of a cluster of size $\{n+1\}$ or a cluster of size $\{n\}$ plus one disconnected atom. With notations introduced in the previous section, the generic expression of this variation of energy writes:

$U_{n+1}^{i}-\left(U_{n}^{j}+U_{1}\right)=\Delta U_{n+1}^{i}-\Delta U_{n}^{j}=\frac{1}{2} W_{a b}\left[N_{a b}^{(i)}(n+1)-N_{a b}^{(j)}(n)-Z\right]$

It is important to note that here $U$ refers to the energy of a particular configuration of the considered cluster (namely $i$ and $j$ for the clusters of size $\{n+1\}$ and $\{n\}$ respectively). 
A further simplification can be done by introducing $N_{\text {bonds }}^{\text {cluster }}$, the number of bonds between the cluster and the site involved in the transformation, i.e. the site whose nature is to be changed. From equation (6) it comes:

$$
\Delta U_{n+1}^{i}-\Delta U_{n}^{j}=-N_{b o n d s}^{\text {cluster }} W_{a b}
$$

\subsection{Elementary transition probability}

To associate a transition probability (per unit time) with a difference of energy between states $\{n\}+\{1\}$ and $\{n+1\}$, for the sake of simplicity, the classical kinetic Ising model is adopted. Consider two states, say $i$ and $j$, such that the change of state of the system defined above can be considered as a single event. The energies associated with these two valleys $U_{i}$ and $U_{j}$ are separated by a saddle point of total height $E_{\text {saddle. }}$. In the case $U_{i}=U_{j}=U_{0}$, it is assumed that $E_{\text {saddle }}-U_{0}$ is always equal to the height of the diffusion barrier entering in the diffusion coefficient. Assuming that $E_{\text {saddle }}$ depends on both initial and final states $U_{0}$ is now replaced by the average energy $\left(U_{i}+U_{j}\right) / 2$ and finally the change in energy takes the wellknown form:

$$
\Delta U_{i->j}=\left(U_{i}+U_{j}\right) / 2+\left(E_{\text {diffusion }}-U_{i}\right)=E_{\text {difiusion }}+\left(U_{j}-U_{i}\right) / 2
$$

The symetrical transition probability associated with $\Delta U_{i \rightarrow j}$ writes:

$$
P_{i \rightarrow j}=v \operatorname{Exp}\left(-\frac{U_{j}-U_{i}}{2 k T}\right)
$$

where the frequency $v$ is related to the diffusion coefficient by the relation $v=\lambda D / a^{2}, \lambda$ is a geometrical parameter depending on the crystallographic structure of parameter $a$ (Philibert $1991)$ and $D$ is the diffusion coefficient of a $B$ atom in the pure $A$ matrix of the form: $D=D_{0} \operatorname{Exp}\left(-E_{\text {diffusion }} / k_{B} T\right)$.

This probability should not be directly compared with the last atomic jump probability in kinetic Monte Carlo simulations (KMC) in which atoms diffuse by exchange with a vacancy. Indeed, the "meso-event" described by equation (12) involves several "microevents" at the atomic scale. Then the respective results for the global event may differ or exactly coincide depending on the lattice, the diffusion coefficient model and other choices in particular the description of the saddle point energy. Similarly, equation (11) is not the only acceptable choice for present calculations; for instance choosing a constant $E_{\text {saddle }}$ by analogy to what is often done in KMC simulations (e.g. Soisson and Martin, 2000) is another simple solution which leads to similar results. And if justified, more elaborated choices are certainly possible as in KMC simulations (for a recent review see Bellon, 2003) but should be developed case by case for specific situations.

\subsection{From individual transition probabilities to average interfacial reaction rates}

Applying this transition model to the two levels of energy entering in equation (12) leads to a pair of symmetrical expressions for capture and release of a specific $B$ atom, respectively:

$$
P_{j i}^{0}(\{n+1, i\} \leftarrow\{n, j\}+\{1\})=v \operatorname{Exp}\left(-\frac{U_{n+1, j}-U_{n, i}-U_{1}}{2 k_{B} T}\right)=\left(\frac{\lambda D}{a^{2}}\right) \operatorname{Exp}\left(+N_{\text {bonds }}^{\text {cluster }}\left(\frac{T_{w}}{T}\right)\right)
$$




$$
P_{i j}^{0}(\{n+1, i\} \rightarrow\{n, j\}+\{1\})=v \operatorname{Exp}\left(+\frac{U_{n+1, j}-U_{n, i}-U_{1}}{2 k_{B} T}\right)=\left(\frac{\lambda D}{a^{2}}\right) \operatorname{Exp}\left(-N_{\text {bonds }}^{\text {cluster }}\left(\frac{T_{w}}{T}\right)\right)
$$

Keeping in mind the final goal, i.e. we want to refer to a cluster only through its size, it means that equations (13-14) have to be averaged over all possible transitions starting from all possible initial configurations (only transitions differing by one atom are considered). At this point it is necessary to introduce another feature. As it can be seen in figure 1, a site of the first shell "applying" for capture is likely to be linked to $N_{\text {bonds }}^{\text {shell }}=1$ to $(Z-1)$ sites of the second shell, which probability of occupancy by a $B$ atom is $X^{\text {shell }}$, the local atomic concentration in $B$ atoms in the second shell. Similarly, $N_{\text {bonds }}^{\text {shell }}$ represents the number of bonds between a $B$ atom of a cluster «applying » for release and the first shell, i.e. it represents the number of sites towards the release can take place. Thus, the general form of the capture and release rates writes respectively $\bar{P}_{n, n+1}=\bar{P}_{n, n+1}^{0} X_{n}^{\text {shell }}$ and $\bar{P}_{n+1, n}=\bar{P}_{n+1, n}^{0}$ (the equivalent of $X_{n}^{\text {shell }}$ for the cluster is by definition equal to 1 ) where the capture and release factors $\bar{P}_{n, n+1}^{0}$ and $\bar{P}_{n+1, n}^{0}$ are the quantities to evaluate as generalisation of equations (13-14):

$$
\begin{aligned}
& \bar{P}_{n, n+1}^{0}(\{n+1\} \leftarrow\{n\}+\{1\})=v\left\{\frac{\sum_{j=1} d_{n, j} \operatorname{Exp}\left(-\frac{U_{n, j}}{k_{B} T}\right) \sum_{i=1} N_{b o n d s, i}^{\text {shell }} \operatorname{Exp}-\left(\frac{U_{n+1, i}-U_{n, j}-U_{1}}{2 k_{B} T}\right)}{\sum_{j=1} d_{n, j} \operatorname{Exp}\left(-\frac{U_{n, j}}{k_{B} T}\right)}\right\} \\
& \bar{P}_{n+1, n}^{0}(\{n+1\} \rightarrow\{n\}+\{1\})=v\left\{\frac{\sum_{i=1} d_{n, i} \operatorname{Exp}\left(-\frac{U_{n+1, i}}{k_{B} T}\right) \sum_{j=1} N_{b o n d s, j}^{\text {shell }} \operatorname{Exp}+\left(\frac{U_{n+1, i}-U_{n, j}-U_{1}}{2 k_{B} T}\right)}{\sum_{i=1} d_{n, i} \operatorname{Exp}\left(-\frac{U_{n+1, i}}{k_{B} T}\right)}\right\}
\end{aligned}
$$

There are two ways to consider this pair of equations. The simplest one is to use the fact that by definition they are thermal averages and then can be computed using a Monte-Carlo procedure. With help of equations (13-14), one can write:

$$
\begin{aligned}
& \bar{P}_{n, n+1}^{0}=v\left\langle\sum N_{b o n d s}^{\text {shell }} \operatorname{Exp}+\left(N_{\text {bonds }}^{\text {cluster }}\left(\frac{T_{w}}{T}\right)\right)\right\rangle_{(n)} \\
& \bar{P}_{n+1, n}^{0}=v\left\langle\sum N_{b o n d s}^{\text {shell }} \operatorname{Exp}-\left(N_{\text {bonds }}^{\text {cluster }}\left(\frac{T_{w}}{T}\right)\right)\right\rangle_{(n+1)}
\end{aligned}
$$

Subscripts $(n)$ and $(n+1)$ mean that the averages have to be calculated on ensembles of configurations of size $(n)$ and $(n+1)$ respectively. Indeed, this form is well appropriated for a statistical evaluation of these two functions; this situation will be described in the next section.

To get a tractable analytical solution for small clusters it is better to remark that the denominators of these expressions are the respective partition functions for cluster sizes $n$ and 
$(n+1)$, as defined in equation 7. An additional simplification appears if these expressions are rewritten using notations introduced in equation (8):

$$
\begin{aligned}
& \bar{P}_{n, n+1}^{0}=v\left\{\frac{\sum_{j=1} d_{n, j} \sum_{i=1} N_{\text {bonds }, i}^{\text {shell }} \operatorname{Exp}-\left(\frac{\Delta U_{n+1, i}+\Delta U_{n, j}}{2 k_{B} T}\right)}{\sum_{j=1} d_{n, j} \operatorname{Exp}\left(-\frac{\Delta U_{n, j}}{k_{B} T}\right)}\right\} \\
& \bar{P}_{n+1, n}^{0}=v\left\{\frac{\sum_{i=1} d_{n+1, i} \sum_{j=1} N_{b o n d s, j}^{\text {shell }} \operatorname{Exp}-\left(\frac{\Delta U_{n+1, i}+\Delta U_{n, j}}{2 k_{B} T}\right)}{\sum_{i=1} d_{n+1, i} \operatorname{Exp}\left(-\frac{\Delta U_{n+1, i}}{k_{B} T}\right)}\right\}
\end{aligned}
$$

In both equations the double sum in the numerator runs over all possible transitions between the set of distinct configurations of state $\{n\}$ and the set of all obtainable configurations of state $\{n+1\}$ in the first case, and between the set of distinct configurations of state $\{n+1\}$ and the set of all obtainable configurations of state $\{n\}$ in the second case. To simplify, say that for reason of symmetry (or reversibility) the numerators of these two equations are strictly equal. This can be readily checked for the simplest possible transition, i.e. between states 1 and 2 of the cubic lattice. Starting from the unique configuration of state 1 there are 6 (i.e. Z) ways to form a 2-atoms cluster. But this set of 6 configurations is in fact constituted of 3 distinct configurations (aligned along $<100>$ directions) appearing each twice. The reverse transition consists to go from these 3 distinct configurations of state 2 towards state 1 by releasing a $B$ atom. For each of these 3 configurations, 2 different $B$ atoms can be removed, then again, one can formed 6 (here identical) configurations of the final state.

The generalization of this simple case requires the definition of another characteristic of clusters which is very important to perform the Monte Carlo calculations reported in the next section: the number of $B$ atoms which can be removed from a cluster of size $(n+1)$ such that it results in a cluster of size $n$ defines the degeneracy factor $D g(n+1)$. In the capture process described by equation (19), the new atom can necessarily be removed without breaking down the cluster, thus for a given configuration of size $(n+1)$ the degeneracy factor $D g(n+1)$ is the number of ways to obtain this configuration from all possible configurations of size $n$. And for the configuration of size $(n+1)$ it is also the number of obtainable configurations of size $n$ (in the simple example depicted above, $D g(2)=2$ ). Therefore, numerators of equations (19) and (20) are only two ways to write the same quantity which will be referred to as the transition function $T_{n, n+1}$ between states $\{n\}$ and $\{n+1\}$ with the remarkable property $T_{n, n+1}=$ $T_{n+1, n}$ :

$T_{n, n+1}=\sum_{j} d_{n, j} \sum_{i} N_{b o n d s, i}^{\text {shell }} \operatorname{Exp}-\left(\frac{\Delta U_{n+1, i}+\Delta U_{n, j}}{2 k_{B} T}\right)$

The advantage of defining this function is that to calculate the capture and release probabilities it is the only function to compute in addition to the (reduced) partition function $Q_{n}^{*}$. The equality $T_{n, n+1}=T_{n+1, n}$ allows for the calculation to be based on the capture mechanism, avoiding the complexity of the release mechanism. Finally, with Eqns. (8, 19-21) the capture and release probabilities write respectively: 


$$
\begin{aligned}
& \bar{P}_{n, n+1}=v\left[\frac{T_{n, n+1}}{Q_{n}^{*}}\right] X_{n}^{\text {shell }} \\
& \bar{P}_{n+1, n}=v\left[\frac{T_{n, n+1}}{Q_{n+1}^{*}}\right]
\end{aligned}
$$

The ratio of these two probabilities takes a form similar to a well-known equation in classical cluster dynamics (equation A6):

$$
\frac{\bar{P}_{n, n+1}}{\bar{P}_{n+1, n}}=\left[\frac{Q_{n+1}^{*}}{Q_{n}^{*}}\right] X_{n}^{\text {shell }}=X_{n}^{\text {shell }} \operatorname{Exp}-\left(\frac{F_{n+1}-F_{n}-F_{1}}{k T}\right)
$$

When the gas of clusters is at equilibrium, $X^{\text {shell }}=X_{1}$ (the solute concentration in the matrix) whatever the size of clusters, then these probabilities verify the "detailed balance". However, using $X^{\text {shell }}$ instead of $X_{1}$ as in the classical scheme is likely to lead to different dynamical behaviour. Indeed, instead of implicitly assuming the interface area around a cluster being at equilibrium, with present coefficients, the system has to couple a local equilibrium and a global equilibrium by adjusting the concentration in the second shell. As a consequence, this concentration, normalized by the monomer concentration in the matrix, is expected to be a function of both cluster size and time, as obtained by Kelton (2000).

\section{§ 4. EXACT SOLUTION FOR SMALL SIZE CLUSTERS}

Starting from the 1-atom cluster (the first parent configuration), the list of its first $Z$ neighbours is built. Then for each member of this list, a 2-atoms cluster (one of the $Z$ child configurations) is built and compared with the list of validated 2-atoms clusters. If it is found to be a copy of a configuration already present in the list it contributes only to $T_{12}$, not to $Q_{2}^{*}$ otherwise a new entry is appended to the list of 2-atoms clusters. The energy and the number of first neighbours are noted for each validated configuration. In addition, to compute the function $T_{n, n+1}$ it is also necessary to note the energy of its parent and $N_{b o n d s}^{\text {shell }}$. As most of the computing time is spent in the comparison of newly formed configurations with the list in construction, some basic tests are performed to save time: the dimensions of the enclosing box, the energy level and the number of first neighbours are first compared with values already accepted. If all these tests are positive, the only safe way to check if the current configuration is a new one or not is to compare this configuration to the referred one, atom per atom. Finally all configurations are arranged according to their level of energy. The same operations are then applied to the next value of $n$, the number of $B$ atoms in the cluster, i.e. for each parent configuration all possible child configurations are built and examined. Due to the exponential growth of the total number of configurations (see the last column in table 1), on a common workstation this exhaustive method can be applied only up to $n=9$ for the cubic structures. One more value can be reasonably obtained but except maybe with massive computing resources the following values will remain out of reach still for some time. Actually even if it would be technically possible to compute few more values, the effort is questionable. It is more efficient to develop a robust Monte-Carlo algorithm with help of the present values as guidelines.

Three quantities have been calculated for the cubic structures as functions of the energy ratio $\left(T_{w} / T\right)$, independently of individual values of bond energies and the temperature: the 
partition functions $Q_{n}^{*}$ (table 1), the number of sites in the first shell $N s h_{1}$ (table 2) and the transition function $T_{n, n+1}$ (table 3 ). The same calculation can also provide the number of sites in the second shell and other quantities if necessary. The average energy can be easily obtained from the expression of $Q_{n}^{*}$.

Coefficients $d_{n, \mathrm{k}}$ found in tables (1-3) can be used to express results as polynomial series of the factor $\varphi=\operatorname{Exp}\left(T_{w} / T\right)$, a common form in the Ising models literature (Domb 1974):

$Q_{n}^{*}=\sum_{k} d_{n, k}^{\text {Table } 1} \varphi^{2(n-1+k-1)}$

$N \operatorname{sh}_{1}(n)=\sum_{k} d_{n, k}^{\text {Table } 2}\left(d_{n, k}^{\text {Table } 1} \varphi^{2(n-1+k-1)}\right) / Q_{n}^{*}$

$T_{n, n+1}=\sum_{k} d_{n, k}^{\text {Table } 3} \varphi^{2(n-1)+k}$

In table 1 , for a given state (line) the levels of energy (columns) are arranged from the left to the right in the decreasing order; this also corresponds to decreasing numbers of distinct configurations. One can see that the numbers of the lowest energy configurations do not follow any logic progression, contrarily to the total number of configurations (column "total") which increases very quickly. This particularity explains the sudden deviations observed at low temperature in MC calculations (see next section) while all results exhibit smooth variations at high or even medium temperature.

- tables 1-3 about here -

\section{§ 5. Monte CARLo CALCUlations FOR MEDiUM SIZE CLUSTERS}

\subsection{Calculation principles}

For cluster sizes larger than 9, Monte Carlo calculations were performed to determine as accurately as possible the numbers of sites in the first and second shells, as well as the capture and release probabilities. According to the present model, if one only needs to know the values of the exchange rates at a given temperature and in a limited range of cluster size (typically 1-100), the free energy itself is no longer necessary as in classical models. But like for exact calculations, available computing power necessary limits the cluster size for which such calculations can be performed in a reasonable amount of time. Beyond this size it is necessary to extrapolate Monte Carlo results, this is where the knowledge of the ratio $Q_{n+1}^{*} / Q_{n}^{*}$ (from which the free energy can be deduced), which can be readily obtained as the ratio $\left(\bar{P}_{n, n+1}^{0}, \bar{P}_{n+1, n}^{0}\right)$, becomes very helpful. Furthermore the knowledge of this quantity can provide the free surface energy, a quantity directly utilisable by classical approaches. Another reason to calculate this quantity for the cubic structure was the possibility to compare with the pioneering work of Jacucci et al. (1983) and Perrini et al. (1984a,b). Using the Monte-Carlo technique of "overlapping distributions" (Bennett 1976) to compute the free energy of clusters up to 20 units (20 solute atoms if one considers segregation) in a 3D cubic lattice, for the first time these authors were able to provide reliable input data for cluster dynamics. In practice, this method is based on the sampling of two functions: the number of $A, B$ atoms linked to the cluster by a number $N b$ of bonds. For small clusters the value $N b=1$ is the only possible choice, then other values become possible as the cluster size increases. Bennet (1976) indicated how to obtain optimal results when several values of $\mathrm{Nb}$ are available but Perrini et al. (1984a,b) did not find any advantage in using larger values of $N b$ for this problem, thus only the value $N b=1$ was used here to compare with results obtained by these authors. In the general case, with present notations, the key equation of this method writes: 


$$
\left(\frac{Q_{n+1}^{*}}{Q_{n}^{*}}\right)=\operatorname{Exp}\left[-\left(Z-2 N_{b}\right)\left(\frac{T_{w}}{T}\right)\right] \frac{\left\langle B_{n+1}^{N_{b}}\right\rangle}{\left\langle A_{n}^{N_{b}}\right\rangle}
$$

where $\left\langle B_{n+1}^{N_{b}}\right\rangle$ and $\left\langle A_{n}^{N_{b}}\right\rangle$ are respectively the number of $B$ and $A$ atoms linked to the cluster by $\mathrm{Nb}$ bonds; the term $(\mathrm{Z}-2 \mathrm{Nb})$ can be derived from equation (6). The complete algorithm to compute $\bar{P}_{n, n+1}^{0}$ and $\bar{P}_{n+1, n}^{0}$ is rather complex and will be described elsewhere. Only the general principles of these MC calculations is briefly indicated, knowing that the basic principle is now very classical: the space of configurations for a given size of cluster is explored by exchanging a $B$ atom with an $A$ atom. In the present work only useful transitions (i.e. such that the result of a permutation of atoms $A$ and $B$ is a cluster of size $n$ ) are considered. No other restriction was imposed in the choice of the pair of $A-B$ atoms, i.e. this process should not be confused with the true release and capture events described by equations (19-20). The transition is accepted according to a Glauber criterion based on the change of energy of the cluster due to its change of shape (cf. equation 6). For a cluster of size $n, n$ successive iterations define a Monte Carlo step per site (MCS). The six functions mentioned above are evaluated every Lmcs MCS, such that Lmcs is larger or equal to 1 to avoid correlations between successive configurations. Using Lmcs $=10$ gives an accuracy approximately three times better than with $L m c s=1$ for a computing time approximately 6 times larger. Between $2 n 10^{5}$ and $10 n 10^{5}$ iterations were performed each run depending on $\mathrm{Lmcs}$ and $(\mathrm{Tw} / \mathrm{T})$.

Comparing with exact values at small cluster sizes, the accuracy of calculations was estimated from the dispersion of more than 50 runs. Best results were obtained for $N s h_{1}$, due to its limited range of variation: about $10^{-4}$, and only weakly dependent on temperature. The least accurate results were obtained for $\left\langle B_{n+1}^{1}\right\rangle$, a quantity of small magnitude but important dispersion and sensible to the temperature: from $210^{-3}$ at $(T w / T) \leq 1.25$ to $210^{-2}$ at $(T w / T)=2$. Low temperatures require special care due to the risk of over-sampling configurations of lowest energies, otherwise the increasingly large real deviations appearing when temperature decreases could also induce important numerical errors. Nevertheless it is impossible to prevent a severe drop of the accuracy at low temperature, except by averaging at least 10 runs. This was not found justified because when the accuracy starts to seriously decrease, for $(T w / T) \geq 1.25$, the ratio between the respective magnitudes of real variations and numerical fluctuations increases quickly from few units up to 10 or more at $(T w / T)=2$; thus curiously, the influence of numerical errors decreases while the accuracy decreases too.

The accuracy of the ratio $Q_{n+1}^{*} / Q_{n}^{*}$ being the sum of relative errors on either $\left\langle A_{n}^{1}\right\rangle$ and $\left\langle B_{n+1}^{1}\right\rangle$ or $\bar{P}_{n, n+1}^{0}$ and $\bar{P}_{n+1, n}^{0}$ is by consequent better with the latter method than with the "overlap distribution" method by a factor 2 approximately, whatever the temperature, due to the relatively poor estimation of $\left\langle B_{n+1}^{1}\right\rangle$. Based on the estimation of $\bar{P}_{n, n+1}^{0}$ and $\bar{P}_{n+1, n}^{0}$, the accuracy of the ratio $Q_{n+1}^{*} / Q_{n}^{*}$ was found better than $10^{-3}$ at high temperature but only of 1.5 $10^{-2}$ for the lowest temperature, which is still convenient. Extensive analysis of results for $n=50$ did not reveal any trend of the accuracy with the cluster size, compared to its temperature sensibility, the accuracy of the algorithm used in this work appears to be independent on the cluster size.

\subsection{Extrapolation of $M C$ results for large size clusters}

Computations have been performed for 8 values of $\left(T_{n} / T\right)$ in the range [0.75 - 2.0]; results obtained for $\left(T_{w} / T\right)=0.5$ are so different and believed to be of poor interest for the purpose of precipitation that they will not discussed here. To fix ideas, if one is interested in $\mathrm{L}_{12}$ clusters in aluminium alloys, the main range of interest for $\left(T_{w} / T\right)$ is typically [1.0 -1.5$]$ 
with a possible extension up to 1.75 ; other values have been computed to help the general understanding and in case it would be useful for other materials or other physical properties. For these 8 values of $\left(T_{n} / T\right)$ the calculations has been done up to $n=100$. In addition, for $\left(T_{w} / T\right)$ in the range [0.75 - 1.25], where the accuracy remains stable, two pairs of cluster sizes were also explored: (199-200) and (399-400), to check the validity of predictions based on the main curve (1-100). Generally speaking, the higher the temperature the smoother the obtained curves, whatever the considered quantity. On the contrary, when the temperature decreases, some fluctuations start to appear, first for small cluster sizes and vanish slowly when the size increases. For $(T w / T)=1$, these fluctuations are typically of the same magnitude than the computing ones then they increase slowly up to $(T w / T)=1.25$. For lower temperatures, the magnitude of these fluctuations continues to increase but faster and faster; for $(T w / T)=1.5$ curves exhibit a regular wavy shape which apparently persists while $n$ increases. Finally, for $(T w / T)=2$ huge variations, in particular of the ratio $Q_{n+1}^{*} / Q_{n}^{*}$ (figure 7 ), can be observed. These fluctuations are normal consequences of the discrete nature of the atomic lattice and in present the case of the low value of $Z$ (cf. section 4). For these reasons, results for sizes (399400) at low temperature would be more confusing than useful; to be relevant one should compute a long sequence of consecutive values, instead of only two values. According to the evolution observed from medium temperature toward low temperature this expensive calculation does not seem justified. On the other hand, at high temperature, the size range (1100) seems too short to fully capture the asymptotic behaviour. Indeed, the behaviour at small cluster sizes is a transient regime whose length increases with temperature, which is likely to induce interpretation errors. A consequence of these real fluctuations is that the apparent accuracy of the fitting procedure, i.e. the envelope of residuals (the difference between computed and analytical values) can be much greater than the estimated numerical accuracy.

Reporting raw results of MC calculations as done in Perrini et al. (1984b) for all quantities and temperatures investigated here is of course not possible. In addition to various graphical representations, to be further used, calculation results need to be fitted with simple functions, if possible. As for any data, fitting MC results has to satisfy several conditions, in particular:

1) If the fit results are to be extrapolated to large size clusters, they should be predictive, i.e. a fit built in a range of $n$ values should be able to correctly predict values not taken into account out of this fit range.

2) fit expressions should use a minimum of parameters to limit their non linearity, in particular the variation of these parameters should be as regular as possible so that results can be interpolated at various temperatures between nodes in the explored range.

As already mentioned most curves exhibit a transient regime at low cluster sizes then reach an asymptotic regime. Thus in practice, depending on the required degree of accuracy and simplicity, it might be necessary to provide two fits, one for small cluster sizes (including or not the sizes for which the exact solution is known) and another one for larger clusters. The difficulty for high temperatures arises from the length of the transient stage and also from the fact that semi-empirical laws giving a good description of results from low to medium temperature are not always relevant at high temperature. Fitting results at low temperature faces another difficulty. Indeed, the fluctuations observed along the curves are likely to severely influence the fitting procedure. For $(T w / T)=2$ the relevance of fitting results becomes even questionable in itself. To conclude about this question, the hope to fit any of the quantities computed here with a universal law relevant at all temperatures and all cluster sizes should be abandoned.

Figure 2 demonstrates the efficiency of the fitting procedure used in this work, in particular for the difference of free energy $F(n)-F(n-1)$ following an expression proposed by Perrini et al. (1984b): 


$$
F(n)-F(n-1)=a\left(n^{2 / 3}-(n-1)^{2 / 3}\right)+b\left(n^{1 / 3}-(n-1)^{1 / 3}\right)+c(\operatorname{Ln}(n)-\operatorname{Ln}(n-1))+d
$$

Here, $a, b, c$ and $d$ were considered as adjustable parameters.

- insert figure 2 about here -

\subsection{The effective interaction surface}

As mentioned in section 5.1 the highest accuracy was obtained for $N s h_{1}$ due to its moderate dispersion. Figure 3 and 4 present the variations of $N s h_{1}$ and $N s h_{2}$ respectively, in the range of cluster size (1-100) for all temperatures investigated in this work. These two functions present similar behaviours and only $N s h_{1}$ was analyzed in details. It can be remarked that curves related to the $(T w / T)=1$ and $(T w / T)=1.5$ (the main range of interest for precipitation in aluminium alloys) define a very narrow region. For $(T w / T)=2$, important fluctuations are present but the curve still fluctuates within this region, at least in this range of cluster size.

To compare the evolution of $N s h_{1}$ with the classical approximation used in cluster dynamics, assuming spherical clusters whatever their size, figure 5 shows the same results than figure 3 but extended up to $n=400$ as well as the solutions for cubic and spherical clusters, assuming that clusters have always a stoechiometric composition. In first approximation, $N s h_{1}$ follows the same power law than the cube- or sphere-solutions. However note that all curves are clearly distinct from the sphere-solution although this solution seems to be an asymptote at macroscopic sizes; but an accurate fit of $N s h_{1}$ reveals a more complex reality. Although the dispersion of curves for the different temperatures is very low, fitting accurately $N s h_{1}$ or $N s h_{2}$ to predict their behaviour at large cluster sizes requires to distinguish two regions: low cluster sizes $(\mathrm{n}<30 \pm 5$ ) and larger sizes (tables 4 and 5). Both regions can be easily fitted with the same expression but with different parameter values:

$$
N \operatorname{sh}(n)=\left(a n^{2 / 3}+b n^{1 / 3}+c\right)
$$

The asymptotic behaviour of $N s h_{1}$ for very large clusters is given by the parameter $a$ in equation (30) or $A 2$ in table 4; its evolution with (T/Tw) is plotted in figure 6 . The result could not be guessed from a simple visual examination of figures 3 and 5 .

$$
\begin{aligned}
& \text { - insert figures 3-5 about here - } \\
& \text { - insert tables 4-5 about here - } \\
& \text { - insert figure } 6 \text { about here - }
\end{aligned}
$$

According to figure 6 , the surface of macroscopic clusters is minimum around $(T w / T)=1.125$ for which it is close to that predicted by the sphere-solution, and increases almost up to the value corresponding to the cube-solution when the temperature either increases or decreases. The evolution of $N s h_{2}$ at large cluster sizes (table 5) as well as other quantities to be further discussed confirms the existence of two distinct behaviours in the $(T w / T)$ range $(0.75-2.0)$. At higher temperature, equation (30) seems inappropriate to accurately describe the observed behaviour even for small to medium clusters. For instance, the last point in figure 6 , for $(T w / T)=0.75$, was obtained by fitting the obtained curve only from $n=80$ and including the pairs of data at $(199,200)$ and $(399,400)$. The same procedure applied to $N s h_{2}$ revealed the same trend, i.e. the coefficient of the term in $n^{2 / 3}$ increases and the coefficient of the term in $n^{1 / 3}$ decreases. In fact this apparently odd evolution of $N s h_{1}$ and $N s h_{2}$ results from two different causes. When the temperature increases, although the shape of large clusters is certainly more spherical than at low temperature, an increasing number of 
configurations can include "cavities" in volume or even more probably "quasi cavities" in surface, the energy cost of such defects decreasing. Thus for a given number of solute atoms the effective volume and surface of the cluster increase mostly because of the roughness of the surface. On the contrary, decreasing the temperature drives the cluster composition toward stoechiometry but enhances the contribution of lowest energy configurations whose shape is almost cubic: the best example is the isolated configuration for $n=8$ (cf. table 1) which is a perfect cube. Therefore the increasing surface of large clusters when temperature decreases reflects mostly the evolution of the cluster shape as observed in KMC simulations (Clouet et al., 2004) or in electron microscopy (Marquis and Seidman, 2001) for $\mathrm{L}_{12}$ clusters of $\mathrm{Al}_{3} \mathrm{Sc}$. Imagine that the Markov chain of visited configurations for a given size of cluster during sampling is transformed into a movie, each image being associated with one of these configurations. At low temperature, one would have the impression of watching the frozen image of a cubic cluster with sharp interfaces. On the contrary, at high temperature the impression would be to watch a more spherical cluster but with very diffuse and always fluctuating interfaces, as a consequence of the exploration of a large number of various shaped configurations.

\subsection{The free energy}

The free energy derived from the ratio $Q_{n}^{*} / Q_{n-1}^{*}$ can be directly obtained from the sampling of $\bar{P}_{n, n+1}^{0}$ and $\bar{P}_{n+1, n}^{0}$ (equations 17-18) or alternatively from the simplest sampling of $\left\langle A_{n}^{1}\right\rangle$ and $\left\langle B_{n+1}^{1}\right\rangle$ as already done in Jacucci et al. (1983) and Perrini et al. (1984a,b). Figure 7 presents the obtained results for the eight reported values of $(T w / T)$. The most striking feature of this figure is the behaviour obtained for $(T w / T)=2$ which contrasts with the monotonous behaviour obtained in the range $(0.75-1.25)$. Note that the first peaks are still obvious for $(T w / T)=1.5$ and can be guessed even for $(T w / T)=1.375$. Again, the origin of these peaks explains easily with table 1 . When temperature becomes very low, the probability to find a cluster in a configuration differing from the lowest energy configuration decreases very quickly, i.e. only the last column in table 1 has to be considered. According to equation (25), the energy associated with this configuration (a function of both $n$ and $k$ in table 1) can be very close or very different from the next and the previous ones. Large clusters exhibit a more regular behaviour but fundamentally their behaviour is not different from that of small clusters. In particular, the sudden drops are related to single values of $n$, while the following increase are related to several successive values of $n$, exactly like for small clusters; the periodicity of these inversed peaks increases linearly with $n$, at least while $n<100$.

\section{- insert figure 7 about here -}

The ratio $Q_{n}^{*} / Q_{n-1}^{*}$ can be directly used but for further analysis it is preferable to transform it into a difference of free energy as reported in figure 8; to improve clarity the curve for $(T w / T)=2$ is not reported. The values of the fit parameters are reported in table 6 for two regions, up to $n=40$ for the first one and from $n=30$ for the second one. For $(T w / T)=1.25$ the simplest capillary approximation $F(n)=a^{2} \sigma n^{2 / 3}$ (here $a$ is the lattice parameter and $\sigma$ is the average surface free energy) gives almost the same quality of fit than the more complex equation (29). For $(T w / T)=0.875$ it is still possible to obtain an acceptable fit using equation (29) provided that the value $n=200$ is also accounted for, otherwise the deviation is about $1 \%$ for the last value, which is small in itself but not acceptable to represent the asymptotic behaviour of large clusters. Finally for $(T w / T)=0.75$ the fitting range should be moved toward much larger value of $n$, as it can be guessed from figure 8. For low temperatures real fluctuations are too large to be reduced by any regular fitting function; one has to accept the 
use of an average and regular function disregarding these fluctuations otherwise it is no longer possible to interpolate present results at intermediate temperatures.

\author{
- insert figure 8 about here - \\ - insert table 6 about here -
}

A widely used quantity in macroscopic models is the average surface free energy $\sigma$ defined as the ratio of the free energy and the surface of the equivalent spherical and isotropic cluster containing $n$ solute atoms, i.e. $F(n)=\sigma(36 \pi)^{1 / 3} n^{2 / 3}$. As long as the free energy can be described with only one parameter, i.e. when $(T w / T) \geq 1.125$, this definition of $\sigma$ is a very convenient convention and is given by parameter $A 2$ in table 6 without any ambiguity. If it is well appropriated to describe the behaviour observed at medium temperatures, describing the free energy at $(T w / T)=2$ with only one parameter as done in table 6 amounts to ignore the large fluctuations (cf. figure 5); although, in first approximation, their periodicity increases linearly with the cluster size, their influence on precipitation kinetics is still unknown. On the other hand, at high temperature, due to the need of additional terms to accurately describe $F(n)$ up to rather large cluster sizes, $\sigma$ is only the asymptotic limit of $F(n) /(36 \pi)^{1 / 3} n^{2 / 3}$ for macroscopic clusters. This need of three parameters for $(T w / T) \leq 1$ and the additional need to account for the data at $n=200$ for $(T w / T)=0.875$, as well as the fact that equation (29) describes results for low sizes but with a different set of parameters values, strongly suggest that even at $(T w / T) \leq 1$ the fitting range should be translated towards higher values of $n$ to correctly describe $F(n)$ with only one parameter as at lower temperature. And in this case the obtained value is likely to differ from the reported value of $A 2$ in table 6 by few percents. Keeping in mind these limitations at extreme temperatures, the obtained values for parameter A2 (table 6) were used to derive the average free surface energy of $\mathrm{Al}_{3} \mathrm{Zr}$ and $\mathrm{Al}_{3} \mathrm{Sc}_{12}$ clusters using inter-atomic potential developed by Clouet et al. (2004) then compared with the values reported by these authors following a completely different procedure, briefly summarized here. The surface energies $\sigma_{100}, \sigma_{110}$ and $\sigma_{111}$ of planes $\{100\},\{110\}$ and $\{111\}$ respectively, were first established using the Bragg-Williams approximation which partly accounts for the non stoechiometry of cluster surfaces. Then a Wulff construction was applied to find the equilibrium shape of clusters at a given temperature as well as the average free surface energy. The comparison (figure 9) shows a good convergence between the two estimations for $\mathrm{T}<650 \mathrm{~K}$ but between this temperature and the melting temperature, present results gives $\sigma$ values decreasing more quickly. The convergence at low temperature and the increasing difference at high temperature between the two methods are absolutely not surprising. The method used in Clouet et al. (2004) allows for an effective decrease of $\sigma_{100}$ with temperature to be accounted for (otherwise the obtained curves would be much flatter), but this decrease might be underestimated at high temperatures due to the increasing nonstoechiometry of clusters. In addition, the Wulff construction is well adapted to low temperatures, characterized by the configuration of lowest energy, but at high temperature this procedure is likely to overestimate the contribution of these low energy configurations. On the contrary, at low temperature the two methods necessarily give very similar results, the absolute asymptote at $0 \mathrm{~K}$ being given by a perfectly cubic cluster with $\{100\}$ facets $\left(\sigma_{100}=\right.$ $W a b / 2)$, which leads to $\sigma=(6 / \pi)^{1 / 3} W a b$; for the parameter A2 in table 6 this corresponds to the maximum value of 3 .

- $\quad$ insert figure 9 about here -

Knowing $[F(n)-F(n-1)]$ and $F(1), F(n)$ can be built by recurrence as done in Perrini et al. (1984b). It is important to note that fitting $F(n)$ leads to poor results compared to a direct fit of the effectively computed quantity $[F(n)-F(n-1)]$ (after some transformation) as done 
above. Intuitively, according to the relation between the ratio $Q_{n}^{*} / Q_{n-1}^{*}$ and the exchange rates, a better correlation is expected between $F(n)$ and $N s h_{1}(n)$, than between $F(n)$ and $n^{2 / 3}$. This correlation is shown in figure 10. The obtained curves can be fitted with a good accuracy by the semi-empirical expression, consistent with equations (29-30):

$$
F(n)=a N s h_{1}(n)+b \operatorname{Ln}\left(N s h_{1}(n)\right)+c
$$

The lower the temperature, the more linear the relation between $F(n)$ and $N s h_{1}(n)$ (table 7). Contrary to the evolution of $[F(n)-F(n-1)]$, the fluctuations of $F(n)$ versus $N s h_{l}(n)$ at $(T w / T)=2$ are smooth and of low amplitude which confirms the high correlation between these two quantities. Although a long transient regime can be observed for $(T w / T)=0.75$, it was possible to get an acceptable fit for available data. At high temperatures the evolution of the leading coefficient $(a)$ in table 7 is very regular (figure 11) and is consistent with the fast decrease of $\sigma$ at high temperatures in figure 9. At low temperature it goes through a maximum at $(T w / T)=1.25$ then decreases very slightly as a consequence of the increase of $N s h_{1}$. The same quantity can be obtained combining $\sigma$ and the leading term of $N s h_{1}(n)$ (table 4 and figure 6); figure 11 shows some differences (up to 6\%) between these two estimations but the two curves have the same shape with a maximum at $(T w / T)=1.25$. In addition, at low temperature they both converge toward the absolute asymptote associated with perfectly cubic clusters. When temperature tends toward zero, the entropy contribution to free energy vanishes thus $F(n)$ converges toward the mean energy $\langle E(n)\rangle$ which is always equal to $<N a b>W a b / 2$ (equation 3), therefore if the cluster shape tends toward a cube of faces $\{100\}$, it implies that $N s h_{1}$ and $N a b$ tend toward $6 n^{2 / 3}$ thus the low temperature asymptote in figure 11 is $1 / 2$.

The differences between the two curves in figure 11 show that the asymptotic behaviour of a given function at large cluster size can hardly be estimated from the value of the leading term of the function used to fit it if this one is not limited to a single term as for $[F(n)-F(n-1)]$ at low temperature (table 6$)$. This is not related to the accuracy of the fitting procedure or the choice of the function but to the fact that the value of the leading factor for a given function is necessarily influenced by the secondary terms; this can be easily checked by changing these additional terms in the fitting function. An accurate value of $\sigma$ can be obtained only because $[F(n)-F(n-1)]$ converges quickly with $n$, except when $(T w / T) \leq 1$ approximately, but the derivative of other functions converge more slowly. Although this might be a coincidence, note that in figure 11 the asymptote $1 / 2$ cuts the curves in the $(T w / T)$ range (11.125), which corresponds very well to the bound between high and low temperature behaviours (figure 6).

- insert figures 10-11 about here -

- insert table 7 about here -

\subsection{The exchange rate factors}

It is reminded that the factors $\bar{P}_{n+1, n}^{0}$ and $\bar{P}_{n, n+1}^{0}$ (equations 17-18) are the only calculated quantities in this work which depend on the choice adopted for the elementary transition probability (see section 3.3); note however that their ratio does not depend on this choice, the free energy is a property of clusters, only the kinetic factors can depend on this choice. Note also that these coefficients being dependent on the structure of the second shell, strictly speaking, using the present values of $\bar{P}_{n+1, n}^{0}$ and $\bar{P}_{n, n+1}^{0}$ for $\mathrm{L}_{12}$ precipitates is only an approximation, contrarily to $N s h_{l}(n)$ and $F(n)$ which are fully correct; the influence of this approximation will be examined in a next paper. 
Although $\bar{P}_{n+1, n}^{0}$ (figure 12) is a quantity varying very regularly, no convenient fitting expression was found. It is more efficient to fit $\bar{P}_{n, n+1}^{0}$ (figure 13) then to use the accurate fit of $[F(n)-F(n-1)]$ and equation (24). Concerning the capture factor $\bar{P}_{n, n+1}^{0}$ (figure 13), although fitting each curve in figure 13 was possible, no predictive solution was found unlike for $F(n)$ or $N s h_{1}(n)$. In fact figure 13 seems rather odd, in particular the spectacular evolution from $(T w / T)=1.5$ to $(T w / T)=2$ and like $F(n), \bar{P}_{n, n+1}^{0}$ is better understood when plotted against $N s h_{1}$ (figure 14) instead of $n$. Within this representation, good fits of $\bar{P}_{n, n+1}^{0}$ can be obtained with expression:

$$
\begin{aligned}
\bar{P}_{n, n+1}^{0} / v \operatorname{Exp}(T w / T)=a N s h_{1}(1 & \left.+c \operatorname{Ln}\left(N s h_{1}\right)\right)+b \sqrt{N s h_{1}}+d \\
& \text { - insert figures 12-13 about here - }
\end{aligned}
$$

Thus, in first approximation the relation between $\bar{P}_{n, n+1}^{0}$ and $N s h_{1}$ is linear, like for the free energy. Disregarding the large fluctuations characteristic $(T w / T) \geq 1.5$, the values of parameters $a, b, c$ and $d$ reported in table 8 show that the lower the temperature the better the linear approximation. For $(T w / T)=1.5$ the ratio between minimum and maximum values of a fluctuation is still close to 1 and the curve can be reasonably replaced by the monotonous curve given by equation (32) but for $(T w / T)=2$ this ratio is larger than 2 , thus fitting with equation (31) or any other monotonous function does not make sense. According to tables (13 ), considering $\mathrm{n}=7$ for the maxima and $\mathrm{n}=8$ for the minima, the ratio of the normalized rate factor $\bar{P}_{n, n+1}^{0}$ tends toward $\operatorname{Exp}(2 T w / T) / 22$ when $T$ tends toward $0 \mathrm{~K}$. This order of magnitude seems compatible with the wide oscillations at larger cluster sizes.

To summarize, starting from low temperature, $\bar{P}_{n, n+1}^{0}$ slightly decreases when temperature increases while $\bar{P}_{n+1, n}^{0}$ increases. Accounting for the respective normalization factors applied to figures 12-14 does not change the relative positions of the different curves. But accounting for the thermally activated diffusion coefficient is likely to inverse the order of curves versus $(T w / T)$ in figure 13-14.

\section{- insert figure 14 about here - \\ - insert table 8 about here -}

This (approximately) linear relation between the capture rate factor $\bar{P}_{n, n+1}^{0}$ and $N s h_{1}$ is the equivalent of the linear relation in classical cluster dynamics between the global absorption rate and the surface of the cluster, considered spherical. However, in the present model, both the active surface for capture $N s h_{1}$ and the proportionality factor between $\bar{P}_{n, n+1}^{0}$ and $N s h_{1}$ are functions of the lattice structure and the key parameter $(T w / T)$. In first approximation (the larger the cluster size, the better the approximation), equation (22) can be rewritten as follow:

$$
\bar{P}_{n, n+1} \approx v\left[\lambda_{\mathrm{n}} \operatorname{Exp}\left(\frac{T w}{T}\right)\right] n^{2 / 3} X_{n}^{\text {shell }}
$$

Similarly, using equation (24), the release rate can be rewritten as: 
$\lambda_{\mathrm{n}}$ is here the parameter $a$ in equation (32) and table 8 multiplied by the coefficient of the term in $n^{2 / 3}$ of $N s h_{1}$ (A2 in table 4), i.e. it is the limit for large values of $\mathrm{n}$ of $\bar{P}_{n, n+1}^{0} / n^{2 / 3}$. The pre-factor $\left[\lambda_{\mathrm{n}} \operatorname{Exp}(T w / T)\right]$ is shown in figure 15, in the represented range of $(T w / T)$ it follows a parabolic law with the maximum value at $(T w / T)=1.5$ equal to about 5 times the minimum value at $(T w / T)=0.75$. In the main range of interest, for $(T w / T)$ between 1 and 1.5 , the central value and its variation are about $110 \pm 55$. It should be noted that in other models, including Kelton's work, this pre-factor is constant with temperature.

- insert figure 15 about here -

\section{§ 6. Conclusion}

A self-coherent definition of interfacial reaction rates in binary alloys for which interactions can be described with an atomic potential limited to the first neighbours (or second neighbours for ordered clusters), and exchanges are limited to capture and release of monomers, has been proposed. It does not require the usual arguments needed by classical cluster dynamics to be derived, in particular equation (A6), but it verifies this well-known condition at equilibrium (the only assumption is that the density of monomers is everywhere the same at equilibrium). Decoupling explicitly the interface mechanisms from the long range diffusion as proposed by Kelton (2000) allows for the development of a framework whose key parameter is the ratio $\left(T w / T=W a b / 2 k_{b} T\right)$, for a given lattice structure. Within this framework the complete definition of a cluster includes two shells in addition to its core; the first shell is the effective collecting surface. A transition function $T_{n, n+1}$ has been defined and enters in the definition of both the capture and the release probabilities. The application of present results obtained for the cubic lattice concerns mostly $\mathrm{L}_{12}$ clusters but the main characteristics of the model as well as the numerical methods can be applied to any crystallographic structure.

For small clusters, up to $n=9$, results (the effective surface, the partition and the transition functions) were obtained exactly and expressed as analytical functions of the factor $(T w / T)$. For larger clusters a classical Monte-Carlo sampling technique has been applied to evaluate the number of sites in the two shells and the exchange rates with a high accuracy except at low temperature. The estimation of the difference of free energy $[F(n)-F(n-1)]$ has been performed following two methods: first as already done by Jacucci et al. (1983) and Perrini et al. (1984a,b), and second from the direct estimation of the capture and release factors. It was found that the second method is about twice more accurate than the first one, whatever the size of clusters and the temperature.

The present model stresses the role of the effective cluster surface for interfacial exchanges compared with the usual surface of the equivalent sphere. Its analysis revealed that for a given cluster size it changes with temperature according to two different mechanisms. In At low temperature, the average cluster shape tends to be cubic with sharp interfaces, while at high temperature it tends to be more spherical but with diffuse interfaces. All properties of clusters, as well as their numerical estimation, are highly influenced by these two effects. For instance, at low temperature the estimations of the free surface energy $\sigma$ of $\mathrm{L}_{12}$ clusters of $\mathrm{Al}_{3} \mathrm{Zr}$ and $\mathrm{Al}_{3} \mathrm{Sc}$ are in excellent agreement with those obtained by Clouet et al. (2004) with classical methods, at their best at low temperature, but the present calculations give a much 
faster decrease of $\sigma$ at high temperature. The asymptotic value for large clusters of the prefactor common to the capture and release rates was found to be temperature dependent instead of being constant as in other models.

Except at low temperature, all computed quantities can be fairly well fitted with simple analytical laws, making possible their extrapolation up to large cluster sizes (at least much larger than actually needed to model the first stages of precipitation kinetics) and interpolated at intermediate temperatures (the accuracy of this procedure can always be improved by additional computations at other temperatures). In return this should open the door to the application of cluster dynamics to complex thermal treatments but the price to pay for the end user will be the choice of a relevant inter-atomic potential, exactly as for doing KMC simulations. Generally speaking, this kind of approach cannot pretend to preserve all the richness of KMC simulations Instead it should be considered as a complementary approach or in some cases an alternative for situations difficult to investigate with KMC simulations due to unreasonable computing time, typically low saturated solutions and / or complex thermal pathways.

\section{ACKNOWLEDGMENTS}

This work would not have been possible without the numerous fruitful discussions I benefited during the joint research program "CPR Precipitation" (Arcelor, Alcan - Pechiney, CNRS and CEA). I am especially grateful to G. Martin for his always constructive criticisms and encouraging remarks and to E. Clouet for helpful discussions and his KMC data. 


\section{Appendix A: Classical cluster dynamics}

The aim of this appendix is to introduce the bases of classical cluster dynamics to the reader not familiar with this formalism. For a more complete discussion and applications, see a recent work by Clouet et al. (2005); other examples can be found for instance in Mathon et al. (1997). In its simplest form, only monomers (1-atom clusters) are mobile. The capture of monomers can be decomposed in two steps: (i) long distance diffusion and (ii) interface reactions. In problems controlled by the long range diffusion, the reaction time is neglected compared to the diffusion time which authorizes to encapsulate the two mechanisms in a pair of so-called emission and absorption rates (also called evaporation and condensation rates), namely $\alpha$ and $\beta$. The kinetics of the clusters population is then described by a simple master equation:

$\dot{C}_{n}(t)=\left(\alpha_{n+1}(t) C_{n+1}(t)+\beta_{n-1}(t) C_{n-1}(t)\right)-\left(\alpha_{n}(t)+\beta_{n}(t)\right) C_{n}(t)$

$C_{n}(t)$ is the atomic concentration of the class $n$ (clusters of $n$ solute atoms).

There is almost no discussion in the literature about the definition of $\beta$. Although there are various ways to obtain the expression of $\beta$, basically they all lead to the same result; here only the simplest one is indicated. Assuming that absorption is diffusion controlled, by reference to the simple case of a hollow sphere of radius $R_{n}$ and surface $S_{n}=4 \pi R_{n}^{2}$ embedded in a quasi infinite matrix of atomic concentration $X_{l}(t)$ at its outer limit, it comes:

$\beta_{n}(t)=\frac{D}{\Omega}\left(\frac{S_{n}}{R_{n}} X_{1}\right)(t)$

$\Omega$ is the atomic volume. Physically, $S_{n}$ is related to the interfacial process, i.e. it is the collecting surface, while $R_{n}$ arises from the (long range) diffusion process. On the contrary, the definition of $\alpha$ is not trivial. It is based on the fact that at equilibrium (symbol " $\sim$ ") $\alpha$ has to satisfy the rule of "detailed balance":

$$
\tilde{\alpha}_{n+1} \tilde{C}_{n+1}=\widetilde{\beta}_{n} \tilde{C}_{n}
$$

The equilibrium concentrations are well approximated by a simple expression derived from Frenkel's work (1955):

$$
\tilde{C}_{n} \approx \tilde{C}_{1}^{n} \operatorname{Exp}\left(-\frac{F_{n}-n F_{1}}{k T}\right)
$$

$F_{n}$ is the free energy of a cluster of size $n$. Combining these equations leads to:

$$
\tilde{\alpha}_{n+1}=\frac{D}{\Omega} \frac{S_{n}}{R_{n}}\left[\frac{C_{1} C_{n}}{\tilde{C}_{n+1}}\right] \approx \frac{D}{\Omega} \frac{S_{n}}{R_{n}} \operatorname{Exp}\left(\frac{F_{n+1}-F_{n}-F_{1}}{k T}\right)
$$

Following an argument formulated by Katz and Spaepen (1978) it is well accepted that emission is a property of the cluster and thus $\alpha$ can always be taken equal to its value at equilibrium given by the above equation. Finally the ratio of the two coefficients writes: 
$\left(\frac{\beta_{n}}{\alpha_{n+1}}\right)(t)=X_{1}(t) \operatorname{Exp}-\left(\frac{F_{n+1}-F_{n}-F_{1}}{k T}\right)$

This expression shows the importance of an accurate estimation of the free energy $F_{n}$ in classical cluster dynamics: indeed, it is the only size dependent parameter which contains some information about the material and the temperature. And in practice, using the classical capillary approximation, the problem is most often reduced to the estimation of the macroscopic surface free energy. This relative poorness is the price to pay for the extreme simplicity and versatility of this method.

\section{References}

BELLON, P. 2003 in Thermodynamics, Microstructures and Plasticity, Eds. A. Finel, D. Maziere and M. Veron, Kluwer Academic Publisher, Dordrecht, The Netherlands.

BENNETT, C., 1976, Journal of Computational Physics, 22, 245.

BERTHIER, F., LEGRAND, B., CREUZE, J. and TETOT, R., 2004, J. Electroanal. Chem., 561, 37; 562, 127.

BINDER, K., STAUFFER, D. and MÜLlER-KRUMBAAR, H., 1974, Phys. Rev. B, 10, 3853.

BINDER, K. and STAUFFER, D., 1976, Advances in Physics, 25, 343.

BINDER, K. 1977, Phys. Rev. B, 15, 4425.

CHRISTIEN, F., BARBU, A., 2004, J. of Nuclear Materials, 324, 90.

CLOUET, E., NASTAR, M. and SIGLI, C., 2004, Phys. Rev. B, 69, 064109.

CLOUET, E. 2004, PhD thesis, Ecole Centrale, Paris.

Available online at : tel.csd.cnrs.fr/documents/archives0/00/00/59/74

CLOUET, E., BARBU, A., LAE, L. and MARTIN, G., 2005, submitted to Acta Materiala.

DOMB, C., 1974, in "Phase Transformations and Critical Phenomena", (C. Domb and M.S. Green, Eds), Academic, London, p. 357.

FRENKEL, J., 1955, Kinetic Theory of Liquids, New York, Dover Publications.

JACUCCI, G., PERRINI, A. and MARTIN, G., 1983, J. Phys. A: Math. Gen., 16, 369.

KATZ, J.L. and SPAEPEN, F., 1978, Phil. Mag. B, 37, 137.

KELTON, K., 2000, Acta Mater., 48, 1967

KELTON, K., GREER, A.L. and THOMPSON, C.V., 1983, J. of Chemical Physics, 79, 6261.

LAE, L., 2004, PhD thesis, INPG, Grenoble.

MARQUIS, E.A. and SEIDMAN, D.N., 2001, Acta Mater., 49, 1909.

MARTIN, G., 1978, in "Solid state phase transformations in metals and alloys", Les Editions de Physique, Orsay, France, p. 337.

MARTIN, G., 1990, Phys Rev. B, 41, 2279.

MARTIN, G., to be published.

MATHON, M.H., BARBU, A., DUNSTETTER, F., MAURY, F., LORENZELLI, N. and DE NOVION, C.H., 1997, J. Nucl. Mater., 245, 224.

MAUGIS, P., SOISSON, F. and LAE, L., 2004, to be published in Proceedings of DIMAT 2004, Crakow, Poland.

PERRINI, A., JACUCCI, G. and MARTIN, G., 1984a, Surface Science, 144, 53.

PERRINI, A., JACUCCI, G. and MARTIN, G., 1984b, Phys. Rev. B, 29, 2689.

PHILIBERT, J., 1991, Diffusion and Mass Transport in Solids, Les Editions de Physique, Les Ulis, France.

RAUTIAINEN, T. and SUTTON, A., 1999, Phys Rev. B, 59, 13681.

SOISSON, F. and MARTIN, G., 2000, Phys. Rev. B, 62, 203.

WAGNER, R. and KAMPMANN, R, 1991, in "Phase Transformations in Materials", Materials Science and Technology, R.W. Cahn, P. Haasen and E.J. Krame, Eds., Vol.

5, VCH Weinheim, p. 215.

WAITE, T.R., 1958, J. Chem. Phys., 28, 103. 


\begin{tabular}{|c|r|r|r|r|r|r|r|}
\hline \multicolumn{7}{|c|}{ Cubic structure } \\
\hline $\mathbf{n} / \mathbf{k}$ & $\mathbf{1}$ & $\mathbf{2}$ & $\mathbf{3}$ & $\mathbf{4}$ & $\mathbf{5}$ & $\mathbf{6}$ & \multicolumn{1}{c|}{ Total } \\
\hline $\mathbf{1}$ & 1 & & & & & & 1 \\
\hline $\mathbf{2}$ & 3 & & & & & & 3 \\
\hline $\mathbf{3}$ & 15 & & & & & & 15 \\
\hline $\mathbf{4}$ & 83 & 3 & & & & & 86 \\
\hline $\mathbf{5}$ & 486 & 48 & & & & & 534 \\
\hline $\mathbf{6}$ & 2967 & 496 & 18 & & & & 3481 \\
\hline $\mathbf{7}$ & 18748 & 4368 & 378 & 8 & & & 23502 \\
\hline $\mathbf{8}$ & 121725 & 36027 & 4854 & 306 & & 1 & 162913 \\
\hline $\mathbf{9}$ & 807381 & 288732 & 51030 & 5544 & 159 & 24 & 1152870 \\
\hline
\end{tabular}

Table 1. $d_{n, k}$ coefficients to compute the reduced partition function $Q_{n}^{*}$ (equation (25)).

\begin{tabular}{|l|c|c|c|c|c|c|}
\hline \multicolumn{7}{|c|}{ Cubic structure } \\
\hline $\mathbf{n} / \mathbf{1}$ & $\mathbf{1}$ & $\mathbf{2}$ & $\mathbf{3}$ & $\mathbf{4}$ & $\mathbf{5}$ & $\mathbf{6}$ \\
\hline $\mathbf{1}$ & 6.0000 & & & & & \\
\hline $\mathbf{2}$ & 10.0000 & & & & & \\
\hline $\mathbf{3}$ & 13.2000 & & & & & \\
\hline $\mathbf{4}$ & 16.2651 & 16.0000 & & & & \\
\hline $\mathbf{5}$ & 19.2840 & 18.5000 & & & & \\
\hline $\mathbf{6}$ & 22.2952 & 21.1855 & 20.6667 & & & \\
\hline $\mathbf{7}$ & 25.2961 & 23.9945 & 23.1111 & 22.0000 & & \\
\hline $\mathbf{8}$ & 28.2847 & 26.8759 & 25.7231 & 24.6667 & & 24.0000 \\
\hline $\mathbf{9}$ & 31.2617 & 29.7883 & 28.4755 & 27.2338 & 26.4528 & 26.0000 \\
\hline
\end{tabular}

Table $2 . d_{n, k}$ coefficients to compute the number of sites in the first shell $N s h_{l}$ (equation (26)).

\begin{tabular}{|c|c|c|r|r|r|r|r|r|}
\hline \multicolumn{7}{|c|}{ Cubic structure } \\
\hline $\mathbf{k} / \mathbf{n}$ & $\mathbf{1}$ & $\mathbf{2}$ & $\mathbf{3}$ & \multicolumn{1}{|c}{} & \multicolumn{1}{|c|}{$\mathbf{5}$} & \multicolumn{1}{c|}{$\mathbf{1}$} & $\mathbf{7}$ & \multicolumn{1}{c|}{$\mathbf{8}$} \\
\hline $\mathbf{1}$ & 30 & 126 & 738 & 4638 & 30528 & 207378 & 1439588 & 10109808 \\
\hline $\mathbf{2}$ & & & 48 & 504 & 4008 & 29808 & 218868 & 1603470 \\
\hline $\mathbf{3}$ & & & & 168 & 2988 & 33924 & 327684 & 2935506 \\
\hline $\mathbf{4}$ & & & & & 240 & 4104 & 45816 & 438626 \\
\hline $\mathbf{5}$ & & & & & & 1260 & 28260 & 390408 \\
\hline $\mathbf{6}$ & & & & & & 72 & 3240 & 56046 \\
\hline $\mathbf{7}$ & & & & & & & 552 & 23460 \\
\hline $\mathbf{8}$ & & & & & & & 0 & 2016 \\
\hline $\mathbf{9}$ & & & & & & & 24 & 456 \\
\hline $\mathbf{1 0}$ & & & & & & & & 0 \\
\hline $\mathbf{1 1}$ & & & & & & & & 72 \\
\hline
\end{tabular}

Table 3. $d_{n, k}$ coefficients to compute the transition function $T_{n, n+1}$ (equation (27)). 


\begin{tabular}{|c|c|c|c|c|c|c|}
\hline $\mathbf{( T w} / \mathbf{T})$ & $\mathbf{A 1}$ & $\mathbf{B 1}$ & $\mathbf{C 1}$ & $\mathbf{A 2}$ & $\mathbf{B 2}$ & $\mathbf{C 2}$ \\
\hline 0.875 & 5.006 & 8.501 & -9.88 & 5.1647 & 5.3786 & -0.97 \\
\hline 1.000 & 4.483 & 8.661 & -8.71 & 4.9837 & 4.9680 & -1.98 \\
\hline 1.125 & 4.534 & 7.171 & -6.49 & 4.9734 & 4.2771 & -1.75 \\
\hline 1.250 & 4.729 & 5.595 & -4.54 & 5.0536 & 3.4886 & -1.15 \\
\hline 1.375 & 4.946 & 4.259 & -3.05 & 5.2150 & 2.5098 & -0.27 \\
\hline 1.500 & 5.206 & 2.903 & -1.63 & 5.3414 & 2.0108 & -0.29 \\
\hline 2.000 & 5.864 & -0.107 & +1.31 & 5.7654 & 0.3954 & +0.35 \\
\hline
\end{tabular}

Table 4: Fit parameters for $N s h_{l}$ with equation (30). First and second sets are convenient for $\mathrm{n}<40$ and $\mathrm{n}>35$, respectively.

\begin{tabular}{|c|c|c|c|c|c|c|}
\hline $\mathbf{( T w} / \mathbf{T})$ & $\mathbf{A 1}$ & $\mathbf{B 1}$ & $\mathbf{C 1}$ & $\mathbf{A 2}$ & $\mathbf{B 2}$ & $\mathbf{C 2}$ \\
\hline 0.875 & 1.2628 & 41.4585 & -35.58 & 4.6741 & 17.6958 & 5.824 \\
\hline 1.000 & 2.3904 & 31.0168 & -19.18 & 4.6680 & 16.6951 & 3.270 \\
\hline 1.125 & 3.4003 & 23.9055 & -9.67 & 4.7766 & 15.6034 & 2.782 \\
\hline 1.250 & 4.2825 & 18.4781 & -2.90 & 4.9079 & 14.8083 & 2.431 \\
\hline 1.375 & 4.6440 & 16.5143 & -1.10 & 5.2418 & 12.7474 & 4.831 \\
\hline 1.500 & 4.8937 & 15.4403 & -0.49 & 5.6280 & 10.5026 & 7.654 \\
\hline 2.000 & 4.9590 & 14.9827 & +0.21 & 7.0228 & 1.86919 & 20.98 \\
\hline
\end{tabular}

Table 5: Fit parameters for $N s h_{2}$ with equation (30). First and second sets are convenient for $\mathrm{n}<40$ and $\mathrm{n}>35$, respectively.

\begin{tabular}{|c|c|c|c|c|c|c|}
\hline $\mathbf{( T w / T )}$ & $\mathbf{A 1}$ & $\mathbf{B 1}$ & $\mathbf{C 1}$ & $\mathbf{A 2}$ & $\mathbf{B 2}$ & $\mathbf{C 2}$ \\
\hline 0.875 & 2.082 & 3.367 & -2.379 & 2.236 & 0.860 & -0.717 \\
\hline 1.000 & 2.305 & 3.312 & -1.980 & 2.446 & 1.427 & -0.9393 \\
\hline 1.125 & 2.466 & 3.019 & -1.617 & 2.697 & $\approx 0.0$ & $\approx 0.0$ \\
\hline 1.250 & 2.580 & 2.744 & -1.362 & 2.800 & 0.0 & 0.0 \\
\hline 1.375 & 2.549 & 3.769 & -1.765 & 2.870 & 0.0 & 0.0 \\
\hline 1.500 & 2.487 & 4.881 & -2.217 & 2.917 & 0.0 & 0.0 \\
\hline 2.000 & & & & 2.957 & 0.0 & 0.0 \\
\hline
\end{tabular}

Table 6: Fit parameters for $[F(n)-F(n-1)] / W_{a b}$ versus $n$ with equation (29). The first and second sets are convenient for $n \leq 40$ and $n \geq 30$, respectively. Parameter $d$ was set equal to 0 and in the second range parameters $b$ and $c$ are necessary only at high temperatures.

\begin{tabular}{|c|c|c|c|}
\hline $\mathbf{( T w} / \mathbf{T})$ & $\mathbf{a}$ & $\mathbf{b}$ & $\mathbf{c}$ \\
\hline 0.750 & 0.3532 & -8.2484 & 27.388 \\
\hline 0.875 & 0.4298 & -3.8602 & 10.057 \\
\hline 1.000 & 0.4796 & -2.6436 & 5.666 \\
\hline 1.125 & 0.5116 & -2.0836 & 3.969 \\
\hline 1.250 & 0.5275 & -1.6351 & 2.840 \\
\hline 1.375 & 0.5271 & -0.8157 & 0.561 \\
\hline 1.500 & 0.5232 & -0.2618 & -0.746 \\
\hline 2.000 & 0.5126 & +0.5021 & -2.254 \\
\hline
\end{tabular}

Table 7: Parameters for fitting $(F(n) / W a b)$ versus $N s h_{1}(n)$ with equation (31). 


\begin{tabular}{|c|c|r|r|r|}
\hline$(\mathbf{T w} / \mathbf{T})$ & $\mathbf{a}$ & \multicolumn{1}{c|}{$\mathbf{b}$} & \multicolumn{1}{c|}{$\mathbf{c}$} & \multicolumn{1}{c|}{$\mathbf{d}$} \\
\hline 0.750 & 2.5049 & 11.441 & 0.0199 & -24.32 \\
\hline 0.875 & 3.8053 & 4.440 & -0.0164 & -8.78 \\
\hline 1.000 & 4.5328 & 1.676 & -0.0259 & -5.36 \\
\hline 1.125 & 5.6790 & -3.998 & -0.0420 & 2.75 \\
\hline 1.250 & 6.0866 & -3.809 & -0.0448 & 2.30 \\
\hline 1.375 & 6.6842 & -4.945 & -0.0517 & 0.92 \\
\hline 1.500 & 6.9655 & -0.591 & -0.0618 & -21.34 \\
\hline
\end{tabular}

Table 8: Fit parameters for the capture rate factor $\bar{P}_{n, n+1}^{0}$ normalized by $\vee \operatorname{Exp}(T w / T)$ as a function of $N s h_{l}$ following equation (32). 


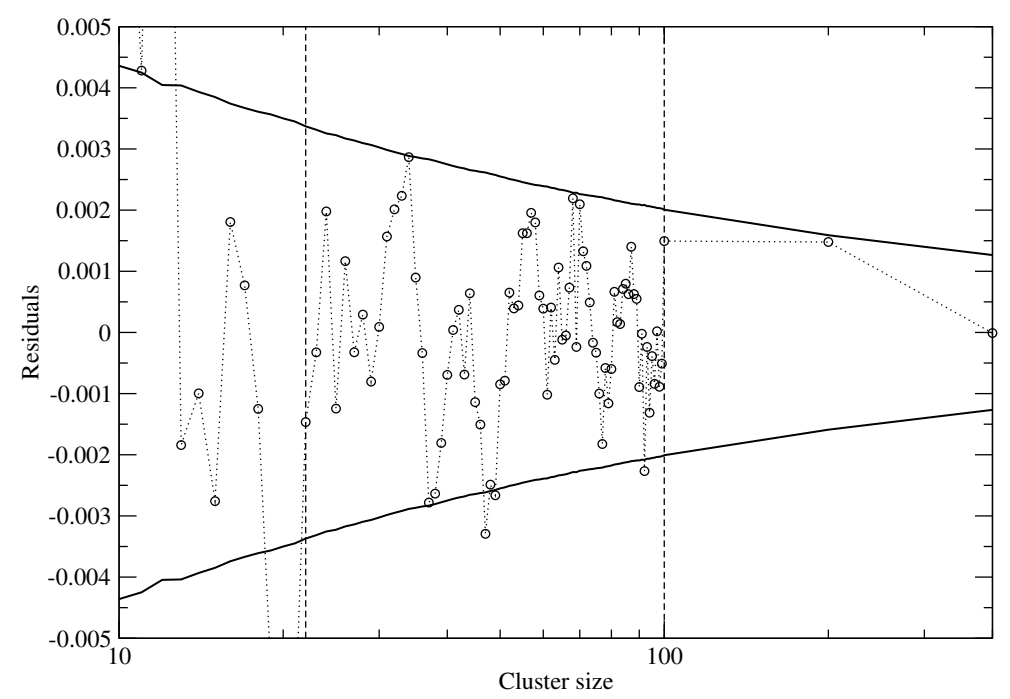

Figure 2: A typical example of the fit procedure applied in this work. The plotted quantity (dotted lines and circles) represents the residuals (i.e. the differences between computed and approximating analytical values) from the fit of $F(n)-F(n-1)$ with equation (29) for $(T w / T)=1.25$, a value for which real fluctuations are still comparable with numerical ones. The pair of convergent curves represents the data to fit multiplied by $\pm 0.5 \%$, a value adjusted to approximately nest the observed deviations in the fitted range from $n=23$ to $n=100$ (vertical dashed lines); the predicted value for $n=400$ is excellent. 


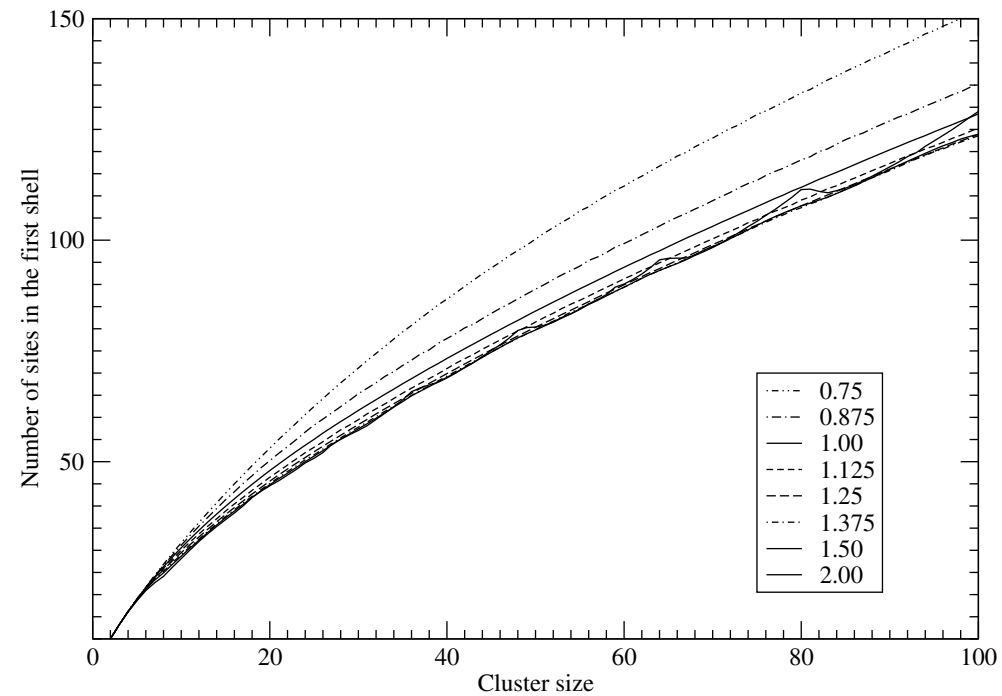

Figure 3: The number of sites in the first shell versus the cluster size for different values of $\left(T_{w} / T\right)$. All curves share the three first points $(6,1),(10,2)$ and $(13.2,3)$ (cf. Table 2$)$.

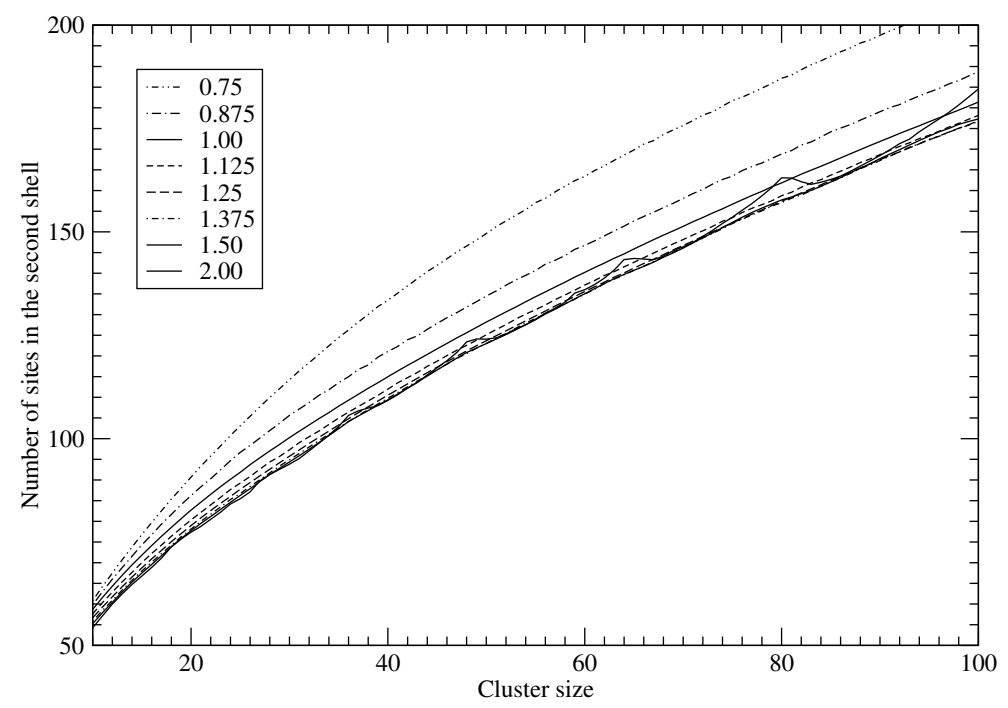

Figure 4: The number of sites in the second shell versus the cluster size for different values of $\left(T_{w} / T\right)$.. 
Figure 5: The global behaviour of the number of sites in the first shell versus the cluster size for different values of $\left(T_{w} / T\right)$. The solutions corresponding to spherical and cubic clusters have been added. The latter is the plain line appearing in second position starting from the bottom left corner or from the upper right corner, crossing most calculated curves in the middle of the figure.

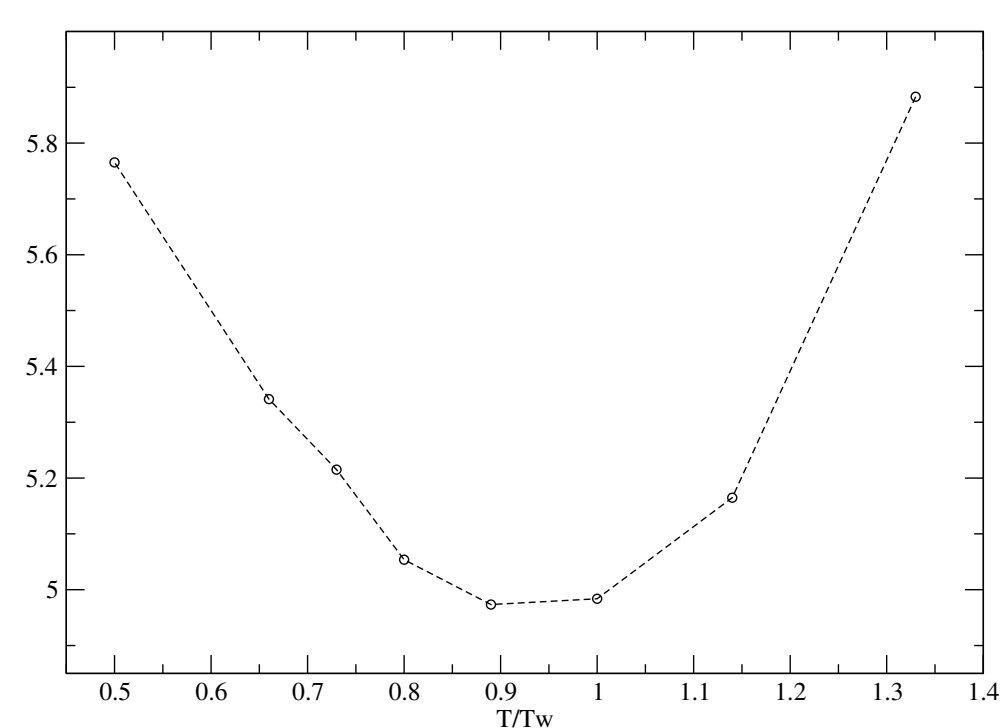

Figure 6: The asymptotic evolution of $N s h_{1}$, i.e. the coefficient of the term $n^{2 / 3}$. The top and the bottom of the figure correspond respectively to a cubic and a spherical cluster (i.e. 6 and $(36 \pi)^{1 / 3}$ ), assumed stoechiometric. 


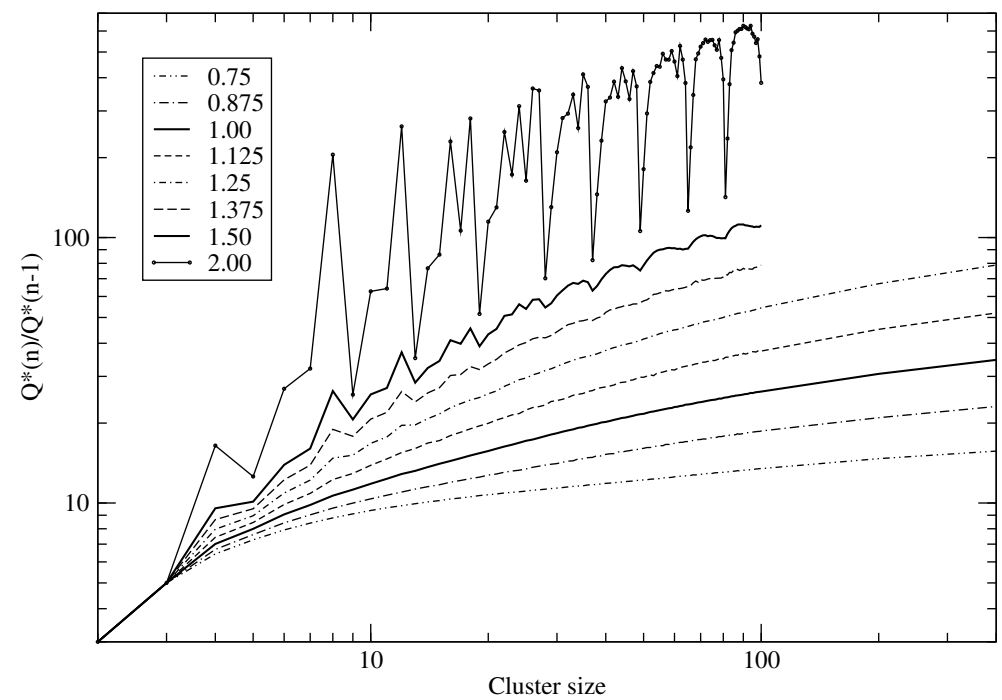

Figure 7: $Q_{n}^{*} / Q_{n-1}^{*}$ versus the cluster size $n$ for different values of $(T w / T)$. Exact values from $n=1$ to $n=9$, MC calculations from $n=10$ to $n=100$ plus $n=200$ and $n=400$ for $(T w / T)$ in the range [0.75 -1.25$]$. The two first values are common to all temperatures.

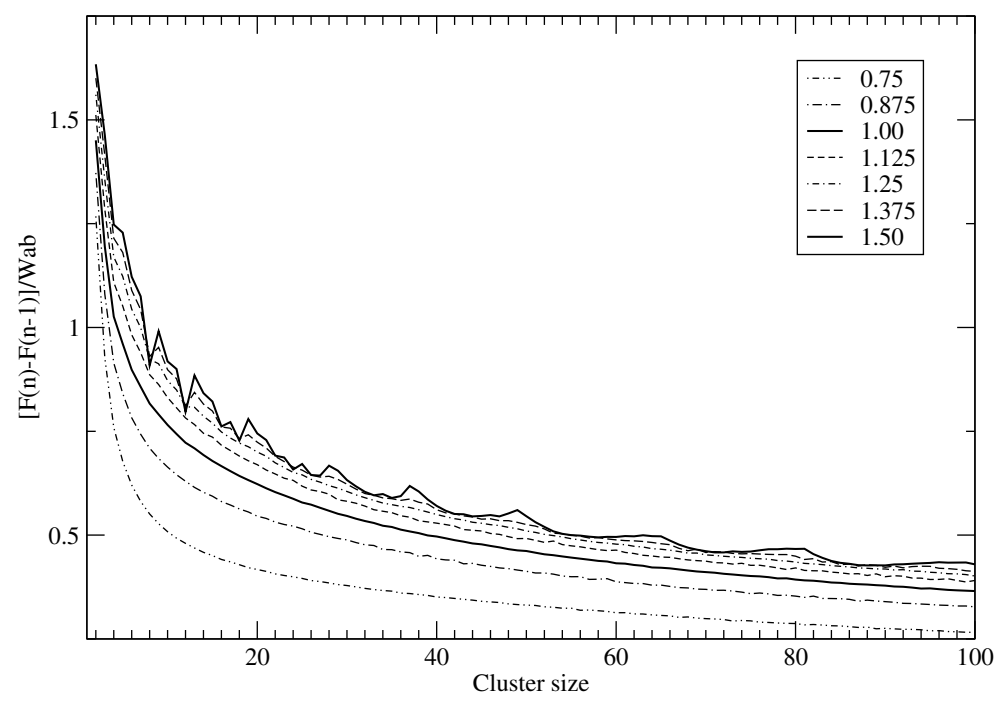

Figure 8: $[F(n)-F(n-1)]$ normalized by $W a b$ versus the cluster size $(n)$ for different values of $(T w / T)$. Exact values for $n=2$ to $n=9$, MC values from $n=10$ to $n=100, n=200$ and $n=400$ in the range $(T w / T)=0.75-1.25$. 


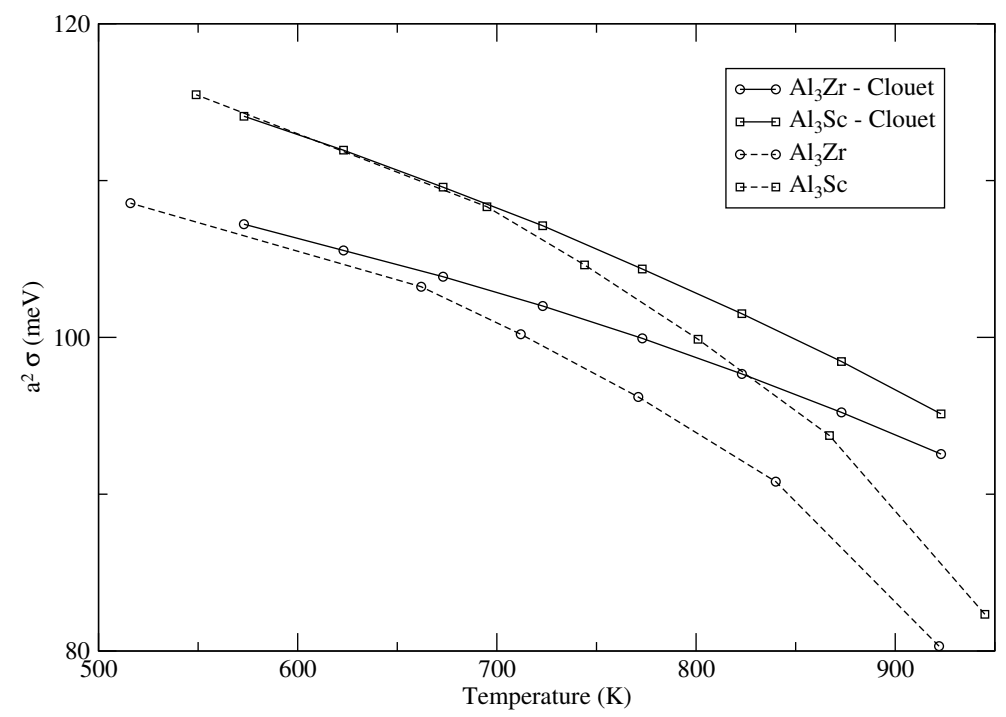

Figure 9: Comparison between the average free surface energy obtained from present results and the values reported by Clouet et al. (2004) for $\mathrm{Al}_{3} \mathrm{Zr}$ and $\mathrm{Al}_{3} \mathrm{Sc} \mathrm{L}_{12}$ clusters. From left to right, plotted points correspond to the $(T w / T)$ range $(2.0-1.0)$ in table 6 (here $a$ is the lattice parameter).

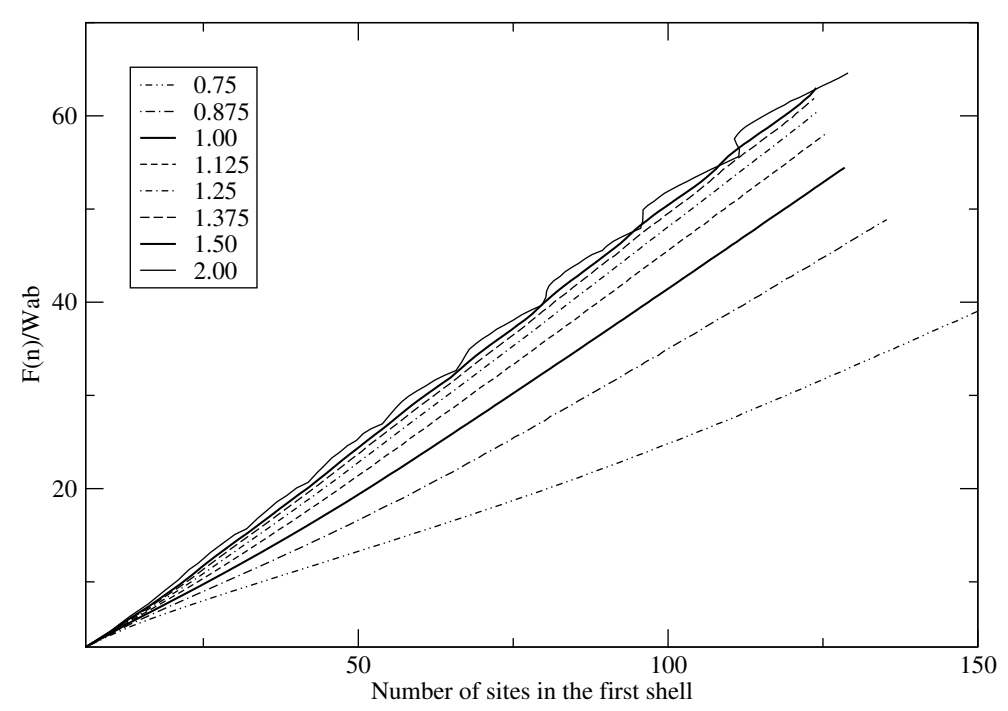

Figure 10: The free energy normalized by $W a b$ versus the number of sites in the first shell for different values of $(T w / T)$, the cluster size varying from 1 to 100 . 


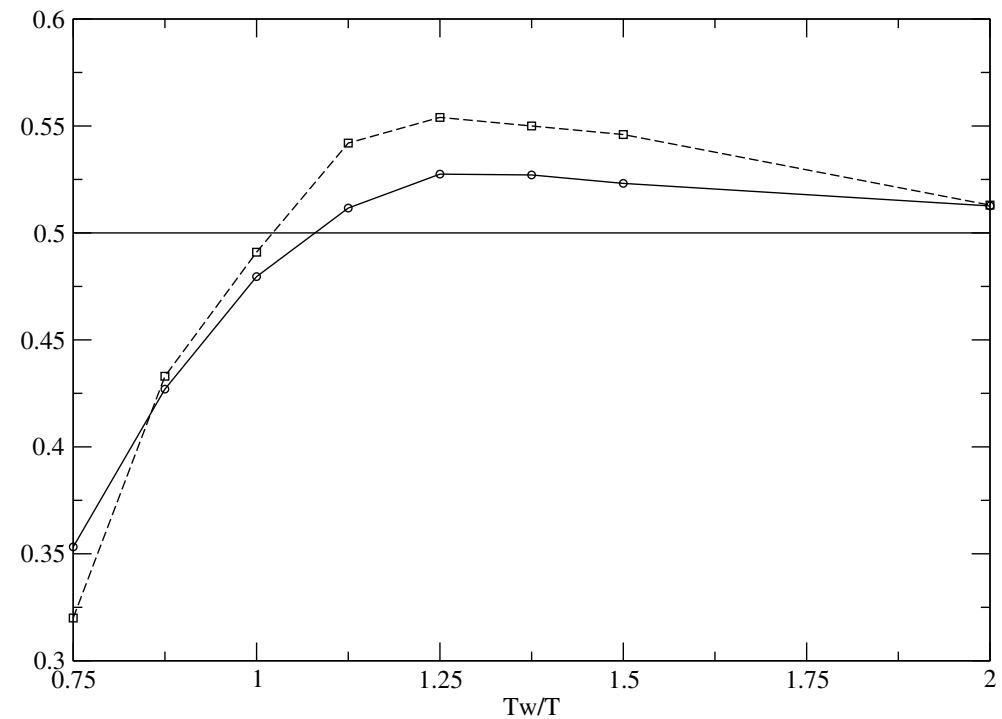

Figure 11: Asymptotic behaviour of $(F(n) / W a b) / N s h_{l}(n)$ for large clusters. Plain curve: parameter $a$ in table 7 . Dashed curve: $\sigma$, i.e. $A 2$ in table 6 , over $A 2$ in table 4 . Plain horizontal line: asymptote at $0 \mathrm{~K}$.

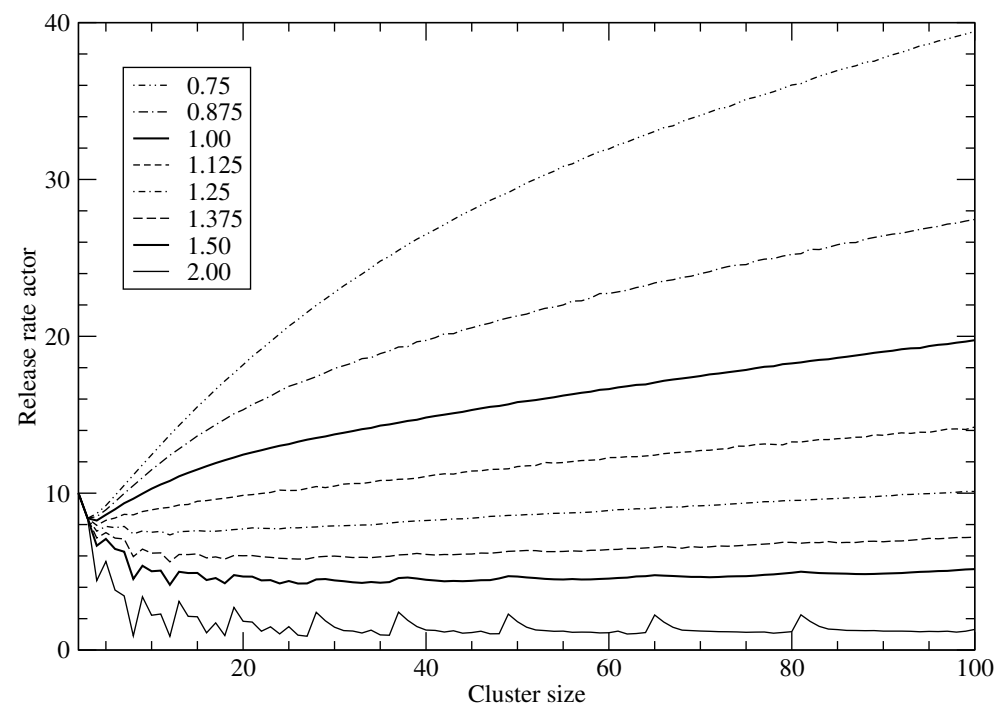

Figure 12: The release rate factor $\bar{P}_{n+1, n}^{0}$ normalized by $\vee \operatorname{Exp}(-T w / T)$ versus the cluster size for different values of $(T w / T)$. The normalized values for $n=2$ and 3 are independent on temperature. 


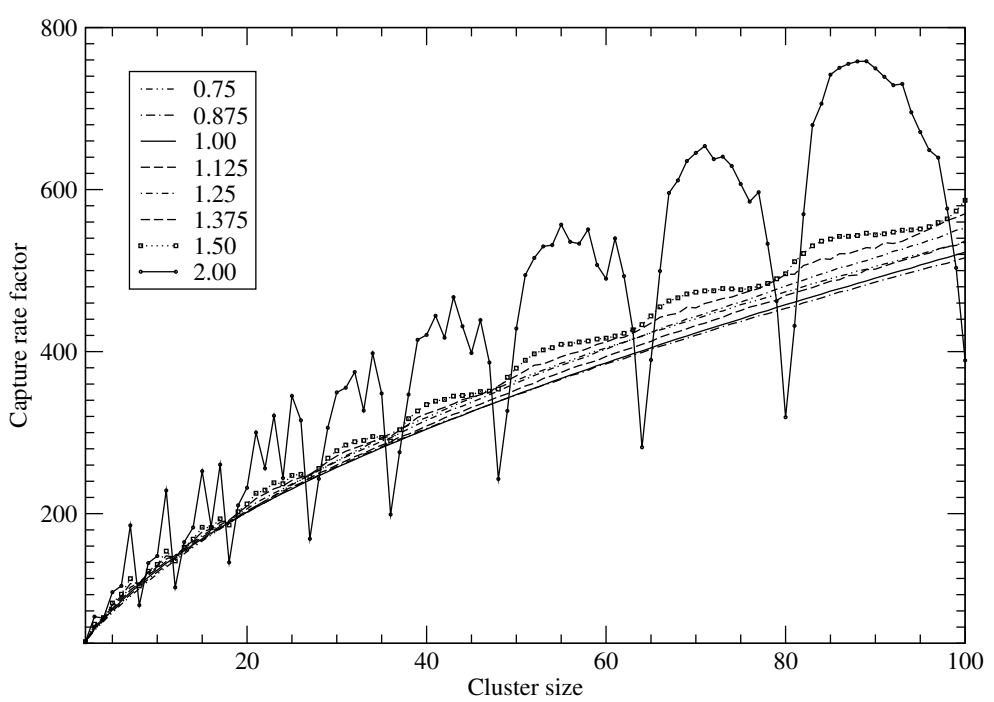

Figure 13: The capture rate factor $\bar{P}_{n, n+1}^{0}$ normalized by $\vee \operatorname{Exp}(T w / T)$ versus the cluster size for different values of $(T w / T)$. The normalized values for $n=2$ and 3 are independent on temperature.

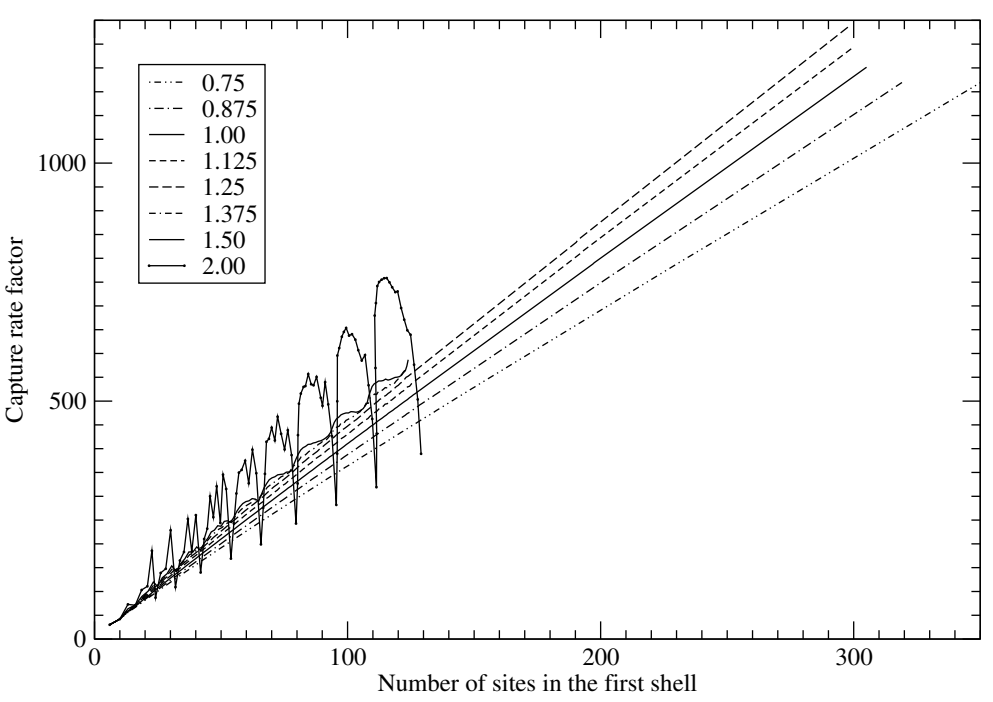

Figue 14 : The capture rate factor $\bar{P}_{n, n+1}^{0}$ normalized by $v \operatorname{Exp}(T w / T)$ versus the number of sites in the first shell for different values of $(T w / T)$, calculated for cluster sizes 1 to 100 for all curves plus (199-200) and (399-400) for the $(T w / T)$ range $(0.75-1.25)$. 


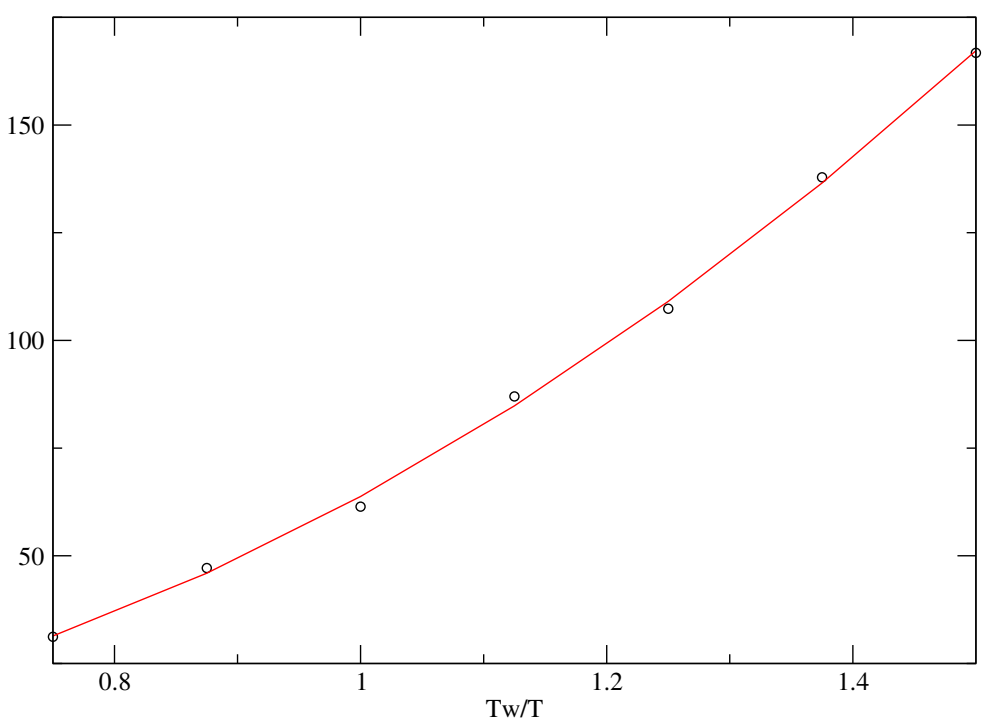

Figure 15: The pre-factor of equations (33-34) as a function of $(T w / T)$. 